\title{
Short proofs of the Kneser-Lovász coloring principle
}

\author{
James Aisenberg a, ${ }^{\mathrm{a}}$, Maria Luisa Bonet ${ }^{\mathrm{b}, 2}$, Sam Buss ${ }^{\mathrm{a}, 1,3}$, Adrian Crăciun ${ }^{\mathrm{c}, 4}$, \\ Gabriel Istrate ${ }^{\mathrm{c}, 4}$

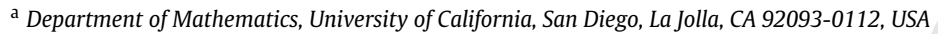 \\ b Computer Science Department, Universidad Politécnica de Cataluña, Barcelona, Spain \\ c West University of Timisoara, and the e-Austria Research Institute, Timisoara, RO-300223, Romania
}

\section{A R T I C L E I N F O}

\section{Article history:}

Received 31 July 2015

Available online $\mathrm{xxxx}$

\begin{abstract}
A B S T R A C T
We prove that propositional translations of the Kneser-Lovász theorem have polynomial size extended Frege proofs and quasi-polynomial size Frege proofs for all fixed values of $k$. We present a new counting-based combinatorial proof of the Kneser-Lovász theorem based on the Hilton-Milner theorem; this avoids the topological arguments of prior proofs for all but finitely many base cases. We introduce new "truncated Tucker lemma" principles, which are miniaturizations of the octahedral Tucker lemma. The truncated Tucker lemma implies the Kneser-Lovász theorem. We show that the $k=1$ case of the truncated Tucker lemma has polynomial size extended Frege proofs.
\end{abstract}

(C) 2018 Published by Elsevier Inc.

\section{Introduction}

This paper discusses proofs of Lovász's theorem about the chromatic number of Kneser graphs and the proof complexity of propositional translations of the Kneser-Lovász theorem. Our main results give a new proof of the Kneser-Lovász theorem, which, for fixed parameter $k$, uses a simple counting argument based on the Hilton-Milner theorem in place of the topological arguments used in prior proofs, for all but finitely many cases. These arguments can be formalized in propositional logic to give polynomial size extended Frege proofs and quasi-polynomial size Frege proofs.

The proof complexity of Frege and extended Frege systems was first studied by Cook and Reckhow [14,15] and Statman [31]. Frege systems (denoted $\mathcal{F}$ ) are sound and complete proof systems for propositional logic with a finite set of schemes for axioms and inference rules. The typical example is a "textbook style" propositional proof system using modus ponens as its only rule of inference. In fact, all Frege systems are equivalent to this system [15]. Extended Frege systems (denoted $e \mathcal{F}$ ) are Frege systems augmented with the extension rule, which allows variables to abbreviate complex formulas. The reader unfamiliar with Frege systems can consult the surveys $[6,11,12,15,24,30]$ for more information.

\footnotetext{
this is an expanded version of a paper [3] which appeared in ICALP 2015.

E-mail addresses: jaisenberg@math.ucsd.edu (J. Aisenberg), bonet@cs.upc.edu (M.L. Bonet), sbuss@ucsd.edu (S. Buss), acraciun@ieat.ro (A. Crăciun), gabrielistrate@acm.org (G. Istrate).

1 Supported in part by NSF grants DMS-1101228 and CCF-1213151. Part of this work was carried out while visiting St. Petersburg with support from the Skolkovo Institute of Technology.

2 Supported in part by grant TIN2013-48031-C4-1.

3 Supported in part by Simons Foundation award 306202.

4 Supported in part by IDEI grant PN-II-ID-PCE-2011-3-0981 "Structure and computational difficulty in combinatorial optimization: an interdisciplinary approach".

https://doi.org/10.1016/j.ic.2018.02.010

0890-5401/C 2018 Published by Elsevier Inc.
}

Please cite this article in press as: J. Aisenberg et al., Short proofs of the Kneser-Lovász coloring principle, Inf. Comput. (2018), https://doi.org/10.1016/j.ic.2018.02.010

() 2018 Elsevier. This manuscript version is made available under the CC-BY-NC-ND 4.0 license http://creativecommons.org/licenses/by-nc-nd/4.0/ 
The size of a Frege or extended Frege proof is the number of symbols in the proof. A proof system $\mathcal{P}_{1}$ simulates a proof system $\mathcal{P}_{2}$ if and only if there is a polynomial $p(n)$ such that, for any propositional formula $\varphi$, if $\varphi$ has a $\mathcal{P}_{2}$-proof of size $n$, then $\varphi$ has a $\mathcal{P}_{1}$-proof of size $\leq p(n)$. Also, $\mathcal{P}_{1}$ quasi-polynomially simulates $\mathcal{P}_{2}$ if and only if there is a $k>0$ such that, if $\varphi$ has a $\mathcal{P}_{2}$-proof of size $n$ then $\varphi$ has a $\mathcal{P}_{1}$-proof of size $\leq 2^{(\log n)^{k}}$. It is trivial that extended Frege systems simulate Frege systems.

It is generally conjectured that the extension rule can provide substantial shortening of proof length, and therefore that Frege systems do not (quasi-polynomially) simulate extended Frege systems. The intuition is that Frege proofs are able to reason using Boolean formulas; whereas extended Frege proofs can reason using Boolean circuits (see [22]). Boolean formulas are conjectured to require exponential size to simulate Boolean circuits. There is no known direct connection to proof complexity, but it is generally conjectured by analogy that there is an exponential separation between the sizes of Frege proofs and extended Frege proofs, and thus that Frege systems do not (quasi-polynomially) simulate extended Frege systems.

Bonet, Buss, and Pitassi [6] systematically looked for combinatorial tautologies that could be candidates for exponentially separating proof sizes for Frege and extended Frege systems. Surprisingly, they found only a small number. The first candidates were based on linear algebra, including the Oddtown theorem, the Graham-Pollack theorem, the Fisher Inequality, the Ray-Chaudhuri-Wilson theorem, and the $A B=I \Rightarrow B A=I$ tautology (the last was suggested by S. Cook). The remaining candidate was Frankl's theorem on the trace of sets. All of these principles were shown to have polynomial size extended Frege proofs, but it was open whether they had polynomial size Frege proofs.

Hrubeš and Tzameret [20] recently showed that the five tautologies based on linear algebra have quasi-polynomial size Frege proofs by showing that there are quasi-polynomial size definitions of determinants whose properties can be established by quasi-polynomial Frege proofs (as was conjectured by [6]). Subsequently, Aisenberg, Bonet, and Buss [2] proved that Frankl's theorem also has quasi-polynomial size Frege proofs. With these results, none of the principles considered by Bonet-Buss-Pitassi provide an exponential separation of Frege and extended Frege systems.

An earlier combinatorial candidate was the pigeonhole principle, introduced by Cook and Reckhow [15]. They showed this has polynomial size extended Frege proofs. Buss [9] later proved this also has polynomial size Frege proofs. Buss's proof was based on "counting", and established that Frege proofs can use polynomial size formulas (based on carry-save addition) to define sizes of sets, and can reason about sizes effectively. Carry-save addition also allows Frege systems to reason about integer multiplication and about adding vectors of integers. The ability of Frege proofs to "count" and to reason about sizes of sets will be important for our Frege proofs of the Kneser-Lovász theorem. The counting proofs were quite different than Cook and Reckhow's inductive proofs of the pigeonhole principle, so these were sometimes taken as evidence that Frege systems do not (quasi-polynomially) simulate extended Frege proofs. However, [7] recently showed that Cook and Reckhow's inductive proofs can be reformulated as quasi-polynomial size Frege proofs.

Another class of candidates is based on consistency statements. We write $\operatorname{Con}_{\mathcal{P}}(n)$ for the propositional statement expressing the condition that the proof system $\mathcal{P}$ does not have a proof of $p \wedge \neg p$ of size $\leq n$. For "natural" systems $\mathcal{P}$ (including Frege and extended Frege systems), the formula $\operatorname{Con}_{\mathcal{P}}(n)$ has size polynomially bounded by $n$ (e.g., [13,10]). Propositional consistency statements have been studied for first-order systems by Pudlák [28,29] and Friedman [unpublished]. Pudlák showed that axiomatizable theories of arithmetic have polynomial size (first-order) proofs of their partial consistency statements; Pudlák and Friedman independently proved polynomial lower bounds as well. Cook [13] showed that an extended Frege system has polynomial size proofs of its own partial consistency statements Con $\mathcal{F}(n)$. Buss [10] proved similarly that a Frege system has polynomial size proofs of its partial consistency statements $\operatorname{Con}_{\mathcal{F}}(n)$.

It also follows from [10] that Frege systems (quasi-)polynomially simulate extended Frege systems iff there are (quasi-)polynomial size Frege proofs of $\operatorname{Con}_{e} \mathcal{F}(n)$. In addition, $\operatorname{Con}_{e} \mathcal{F}(n)$ is a "logical" principle not really a "combinatorial" principle. ${ }^{5}$ For these reasons, partial consistency statements such as $\operatorname{Con}_{\mathcal{F}} \mathcal{F}(n)$ do not serve as the kinds of candidates for separating Frege and extended Frege system that we are seeking.

Other candidates for exponentially separating Frege and extended Frege systems arose from the work of Kołodziejczyk, Nguyen, and Thapen [23] in the setting of bounded arithmetic [8]. These include various forms of the local improvement principles $\mathrm{LI}_{1} \mathrm{LI}_{\log }$ and LLI. The results of [23] showed that the LI principle is many-one complete for the NP search problems of $V_{2}^{1}$; it follows that $\mathrm{LI}$ is equivalent to partial consistency statements for extended Frege systems. Beckmann and Buss [5] subsequently proved that $\mathrm{LI}_{\log }$ is provably equivalent (in $S_{2}^{1}$ ) to $\mathrm{LI}$ and that the linear local improvement principle LLI is provable in $U_{2}^{1}$. The LLI principle thus has quasi-polynomial size Frege proofs. Combining the results of [5,23] shows that $\mathrm{LI}_{\log }$ and LLI are many-one complete for the NP search problems of $V_{2}^{1}$ and $U_{2}^{1}$, respectively, and thus equivalent to partial consistency statements for extended Frege and Frege systems, respectively.

Thus, apart from partial consistency statement, none of the above principles serve as combinatorial candidates for showing that Frege systems do not quasi-polynomially simulate extended Frege systems.

A new candidate based on the Kneser-Lovász theorem was recently proposed by Istrate and Crăciun [21]. As defined below, the Kneser-Lovász theorem gives a lower bound on the chromatic of the $(n, k)$-Kneser graphs. Istrate and Crăciun showed that the $k=3$ case of these tautologies have polynomial size extended Frege proofs, but left open whether they

\footnotetext{
5 However, see Avigad [4] for a combinatorial version of $\operatorname{Con}_{e} \mathcal{F}(n)$.
} 
have (quasi-)polynomial size Frege proofs. However, the main results of the present paper show that, for any fixed $k \geq 1$, the Kneser-Lovász tautologies have quasi-polynomial size Frege proofs. Thus these also do not give an exponential separation of Frege from extended Frege systems.

With these last results, we have few remaining combinatorial candidates for showing Frege systems do not quasipolynomially simulate extended Frege systems. One remaining candidate is tautologies based on the Rectangular Local Improvement principles, $\mathrm{RLI}_{k}$, of Beckmann-Buss [5] for fixed $k \geq 2$. The only other combinatorial candidate we know of is introduced in Section 6 below. This is the $k=1$ case of the "truncated Tucker lemma". Theorem 25 shows it has polynomial size extended Frege proofs; however, we have been unable to show that it has quasi-polynomial size Frege proofs.

The outline of the paper is as follows. First, in Section 2 we define the $(n, k)$-Kneser graphs and state Lovász's theorem about their chromatic numbers. Theorems 4 and 5 state our main results about Frege and extended Frege proofs of that theorem. Section 3 gives an informal ("mathematical") proof of the Kneser-Lovász theorem using a new proof method based on a simple counting argument. Prior proofs used, at least implicitly, a topological fixed-point lemma. The most combinatorial proof is by Matoušek [26] and is inspired by the octahedral Tucker lemma; see also Ziegler [32]. Our new proofs mostly avoid topological arguments and use a counting argument instead. The counting arguments are used to prove the existence of "star-shaped" color classes. These counting arguments can be formalized with Frege proofs. For the Kneser-Lovász theorem, the counting arguments reduce the general case to "small" instances of size $n \leq 2 k^{4}$. For fixed $k$, there are only finitely many small instances, and they can be verified by exhaustive enumeration. As we shall see, this leads to polynomial size extended Frege proofs, and quasi-polynomial size Frege proofs for the Kneser-Lovász principles. Sections 3.1 and 3.2 give two "mathematical" versions of the counting proofs, which will be formalized as extended Frege proofs and Frege proofs (respectively). Section 3.3 is a short diversion and considers whether there are colorings of the Kneser graphs with many non-starshaped color classes.

Section 4 discusses some of the details of formalizing the arguments in Section 3 in the Frege and extended Frege systems, establishing our two main theorems. We focus on expressing the concepts described in Section 3 in propositional logic, and we only sketch some of the details of how Frege systems can prove properties of these concepts.

The proofs of the Kneser-Lovász theorem in Sections 3 and 4 reduce the general case of the Kneser-Lovász theorem to finitely many base cases, which are then handled by exhaustive enumeration. It would be interesting to give a uniform proof that does not need to handle the base cases in this way. Motivated by this, Section 5 defines new "truncated" forms of the octahedral Tucker lemma. These truncated Tucker lemmas can be expressed as families of polynomial size propositional tautologies. The octahedral Tucker lemma, on the other hand, can only be expressed by exponential size formulas. Matoušek showed that the Kneser-Lovász theorem follows from the octahedral Tucker lemma. We refine this by proving that the octahedral Tucker lemma implies the two truncated Tucker lemmas, that the two versions of the truncated Tucker lemma are equivalent, and that the truncated Tucker lemmas imply the Kneser-Lovász theorem. Since the truncated Tucker lemmas can be expressed as polynomial size tautologies, it is natural to ask about their proof complexity in (extended) Frege systems. Section 6 establishes that the $k=1$ cases of the truncated Tucker lemmas have polynomial size extended Frege proofs. It is open whether these have (quasi-)polynomial size Frege proofs. Thus, this is a candidate for separating Frege and extended Frege systems. Likewise, it is open whether the truncated Tucker lemmas for $k>1$ have subexponential size extended Frege proofs. For this, it is tempting to try to modify the combinatorial proof of the Tucker lemma of Freund and Todd [18] (see also Matoušek [26]). Their proof uses a version of the parity principle PPA [27]. In fact, the general (not necessarily octahedral) Tucker lemma is known to be many-one complete for PPA [1]. However, Freund and Todd's proof applies the parity principle to exponentially large graphs, and this prevents us from directly formalizing their arguments with polynomial size extended Frege proofs.

We thank the two referees for helpful comments and suggestions.

\section{The Kneser-Lovász principle and statement of the main theorems}

The $(n, k)$-Kneser graph is defined to be the undirected graph whose vertices are the $k$-subsets of $\{1, \ldots, n\}$; there is an edge between two vertices iff those vertices have empty intersection. The Kneser-Lovász theorem states that Kneser graphs have a large chromatic number:

Theorem 1 (Lovász [25]). Let $n \geq 2 k>1$. The ( $n, k)$-Kneser graph has no coloring with $n-2 k+1$ colors.

It is well-known that the $(n, k)$-Kneser graph has a coloring with $n-2 k+2$ colors (see Section 3.3), so the bound $n-2 k+1$ is optimal. For $k=1$, the Kneser-Lovász theorem is just the pigeonhole principle.

Istrate and Crăciun [21] noted that, for fixed values of $k$, the propositional translations of the Kneser-Lovász theorem are polynomial size in $n$. They presented proofs that can be formalized by polynomial size Frege proofs for $k=2$, and by polynomial size extended Frege proofs for $k=3$. This left open the possibility that the $k=3$ case could exponentially separate the Frege and extended Frege systems. It was also left open whether the $k>3$ case of the Kneser-Lovász theorem gave tautologies that require exponential size extended Frege proofs. As discussed above, the present paper refutes these possibilities. Theorems 4 and 5 summarize these results.

Let $[n]$ be the set $\{1, \ldots, n\}$; members of $[n]$ are called nodes. We identify $\left(\begin{array}{l}n \\ k\end{array}\right)$ with the set of $k$-subsets of [n], the vertices of the $(n, k)$-Kneser graph. 
Definition 2. An m-coloring of the (n,k)-Kneser graph is a map $c$ from $\left(\begin{array}{l}n \\ k\end{array}\right)$ to $[m]$, such that for $S, T \in\left(\begin{array}{l}n \\ k\end{array}\right)$, if $S \cap T=\emptyset$, then $c(S) \neq c(T)$. If $\ell \in[m]$, then the color class $P_{\ell}$ is the set of vertices assigned the color $\ell$ by $c$.

The formulas Kneser $_{k}^{n}$ are the natural propositional translations of the statement that there is no $(n-2 k+1)$-coloring of the $(n, k)$-Kneser graph:

Definition 3. Let $n \geq 2 k>1$, and $m=n-2 k+1$. For $S \in\left(\begin{array}{l}n \\ k\end{array}\right)$ and $i \in[m]$, the propositional variable $p_{S, i}$ has the intended meaning that vertex $S$ of the Kneser graph is assigned the color $i$. The formula $\operatorname{Kneser}_{k}^{n}$ is

$$
\bigwedge_{S \in\left(\begin{array}{l}
n \\
k
\end{array}\right)} \bigvee_{i \in[m]} p_{S, i} \rightarrow \bigvee_{\substack{S, T \in\left(\begin{array}{c}
n \\
k
\end{array}\right) \\
S \cap T=\emptyset}} \bigvee_{i \in[m]}\left(p_{S, i} \wedge p_{T, i}\right)
$$

Theorem 4. For fixed parameter $k \geq 1$, the propositional translations $\operatorname{Kneser}_{k}^{n}$ of the Kneser-Lovász theorem have polynomial size extended Frege proofs.

Theorem 5. For fixed parameter $k \geq 1$, the propositional translations $\operatorname{Kneser}_{k}^{n}$ of the Kneser-Lovász theorem have quasi-polynomial size Frege proofs.

When both $k$ and $n$ are allowed to vary, it is open whether the $\operatorname{Kneser}_{k}^{n}$ tautologies have quasi-polynomial size (extended) Frege proofs, or equivalently, have proofs with size quasi-polynomially bounded in terms of $n^{k}$.

\section{Mathematical arguments}

Section 3.1 gives the new proof of the Kneser-Lovász theorem; this is later shown to be formalizable with polynomial size extended Frege proofs. Section 3.2 gives a slightly more complicated but more efficient proof, later shown to be formalizable with quasi-polynomial size Frege proofs. The next definition and lemma are crucial for Sections 3.1 and 3.2.

Any two vertices in a color class $P_{\ell}$ have nonempty intersection. One way this can happen is for the color class to be "star-shaped":

Definition 6. A color class $P_{\ell}$ is star-shaped if $\bigcap P_{\ell}$ is nonempty. If $P_{\ell}$ is star-shaped, then any $i \in \bigcap P_{\ell}$ is called a central node of $P_{\ell}$.

The intuition is that non-starshaped color classes are too small to cover all $\left(\begin{array}{l}n \\ k\end{array}\right)$ vertices. The Hilton-Milner theorem [19] (which is a refinement of the Erdős-Ko-Rado [16] theorem on intersecting families of finite sets, and improves on Theorem 2(ii) of [16]) implies that if a color class $P_{\ell}$ is not star-shaped then $P_{\ell}$ has size $\left|P_{\ell}\right| \leq 1+\left(\begin{array}{c}n-1 \\ k-1\end{array}\right)-\left(\begin{array}{c}n-k-1 \\ k-1\end{array}\right)$. A simpler proof of their bound was given by Frankl and Füredi [17]. Hilton and Milner also observe that their bound is optimal. Instead of using the Hilton-Milner bound, we state a weakened form as Lemma 7, which has a substantially simpler proof. (By comparison, the Hilton-Milner bound is $\leq k\left(\begin{array}{c}n-2 \\ k-2\end{array}\right)$ for $k \geq 3$.) Lemma 7 will be used in our proof of the Kneser-Lovász theorem to establish the existence of star-shaped color classes.

Lemma 7. Let c be a coloring of $\left(\begin{array}{l}n \\ k\end{array}\right)$. If $P_{\ell}$ is not star-shaped, then

$$
\left|P_{\ell}\right| \leq k^{2}\left(\begin{array}{l}
n-2 \\
k-2
\end{array}\right)
$$

Proof. Suppose $P_{\ell}$ is not star-shaped. If $P_{\ell}$ is empty, the claim is trivial. So suppose $P_{\ell} \neq \emptyset$, and let $S_{0}=\left\{a_{1}, \ldots, a_{k}\right\}$ be some element of $P_{\ell}$. Since $P_{\ell}$ is not star-shaped, there must be sets $S_{1}, \ldots, S_{k} \in P_{\ell}$ with $a_{i} \notin S_{i}$ for $i=1, \ldots, k$.

To specify an arbitrary element $S$ of $P_{\ell}$, we do the following. Since $S$ and $S_{0}$ have the same color, $S \cap S_{0}$ is nonempty. We first specify some $a_{i} \in S \cap S_{0}$. Likewise, $S \cap S_{i}$ is nonempty; we second specify some $b \in S \cap S_{i}$. By construction, $a_{i} \neq b$, so $S$ is fully specified by the $k$ possible values for $a_{i}$, the $k$ possible values for $b$, and the $\left(\begin{array}{l}n-2 \\ k-2\end{array}\right)$ possible values for the remaining members of $S$. Therefore, $\left|P_{\ell}\right| \leq k^{2}\left(\begin{array}{c}n-2 \\ k-2\end{array}\right)$.

\subsection{Argument for extended Frege proofs}

Let $k>1$ be fixed. We prove the Kneser-Lovász theorem by induction on $n$. The base cases for the induction are $n=$ $2 k, \ldots, N(k)$ where $N(k)$ is the constant depending on $k$ specified in Lemma 8. We shall show that $N(k)$ is no greater than $k^{4}$. Since $k$ is fixed, there are only finitely many base cases. Since the Kneser-Lovász theorem is true, these base cases can all be proved by a fixed Frege proof of finite size (depending on $k$ ). Therefore, in our proof below, we only show the induction step. 
Lemma 8. Fix $k>1$. There is an $N(k)$ so that, for $n>N(k)$, any $(n-2 k+1)$-coloring of $\left(\begin{array}{l}n \\ k\end{array}\right)$ has at least one star-shaped color class.

Proof. Suppose that a coloring $c$ has no star-shaped color class. Since there are $n-2 k+1$ many color classes, Lemma 7 implies that

$$
(n-2 k+1) \cdot k^{2}\left(\begin{array}{l}
n-2 \\
k-2
\end{array}\right) \geq\left(\begin{array}{l}
n \\
k
\end{array}\right) .
$$

For fixed $k$, the left-hand side of $(1)$ is $\Theta\left(n^{k-1}\right)$ and the right-hand side is $\Theta\left(n^{k}\right)$. Thus, there exists an $N(k)$ such that $(1)$ fails for all $n>N(k)$. Hence for $n>N(k)$, there must be at least one star-shaped color class.

To obtain an upper bound on the value of $N(k)$, note that (1) is equivalent to

$$
(n-2 k+1) k^{3}(k-1) \geq n(n-1) .
$$

Since $2 k-1 \geq 1,(2)$ implies that $(n-1) k^{4}>n(n-1)$ and thus that $n<k^{4}$. Thus, (1) will be false if $n \geq k^{4}$; so $N(k)<k^{4}$. We are now ready to give our first proof of the Kneser-Lovász theorem.

Proof of Theorem 1, except for base cases. Fix $k>1$. By Lemma 8, there is some $N(k)$ such that for $n>N(k)$, any $(n-2 k+1)$-coloring $c$ of $\left(\begin{array}{l}n \\ k\end{array}\right)$ has a star-shaped color class. As discussed above, the cases where $n \leq N(k)$ are handled by exhaustive search and the truth of the Kneser-Lovász theorem. For $n>N(k)$, we prove Theorem 1 by infinite descent. In other words, we show that if $c$ is an $(n-2 k+1)$-coloring of $\left(\begin{array}{l}n \\ k\end{array}\right)$, then there is some $c^{\prime}$ that is an $((n-1)-2 k+1)$-coloring of $\left(\begin{array}{c}n-1 \\ k\end{array}\right)$.

By Lemma 8 , the coloring $c$ has some star-shaped color class $P_{\ell}$ with central node $i$. Without loss of generality, $i=n$ and $\ell=n-2 k+1$. Let

$$
c^{\prime}=c \uparrow\left(\begin{array}{c}
n-1 \\
k
\end{array}\right)
$$

be the restriction of $c$ to the domain $\left(\begin{array}{c}n-1 \\ k\end{array}\right)$. This discards the central node $n$ of $P_{\ell}$, and thus all vertices with color $\ell$. Therefore, $c^{\prime}$ is an $((n-1)-2 k+1)$-coloring of $\left(\begin{array}{c}n-1 \\ k\end{array}\right)$. This completes the proof.

\subsection{Argument for Frege proofs}

We now give a second proof of the Kneser-Lovász theorem. The proof above required $n-N(k)$ rounds of infinite descent to transform a Kneser graph on $n$ nodes to one on $N(k)$ nodes. Our second proof replaces this with only $O(\log n)$ many rounds, and this efficiency will be key for formalizing this proof with quasi-polynomial size Frege proofs in Section 4.2 .

We refine Lemma 8 to show that for $n$ sufficiently large, there are many (i.e., a constant fraction) star-shaped color classes. The idea is to combine the upper bound of Lemma 7 on the size of non-starshaped color classes with the trivial upper bound of $\left(\begin{array}{l}n-1 \\ k-1\end{array}\right)$ on the size of star-shaped color classes.

Lemma 9. Fix $k>1$ and $0<\beta<1$. Then there exists an $N(k, \beta)$ such that for $n>N(k, \beta)$, if $c$ is an $(n-2 k+1)$-coloring of $\left(\begin{array}{l}n \\ k\end{array}\right)$, then $c$ has at least $\frac{n}{k} \beta$ many star-shaped color classes.

Proof. The value of $N(k, \beta)$ can be set equal to $\frac{k^{3}(k-\beta)}{1-\beta}$. Let $n>\frac{k^{3}(k-\beta)}{1-\beta}$, and suppose $c$ is an $(n-2 k+1)$-coloring of $\left(\begin{array}{l}n \\ k\end{array}\right)$. Let $\alpha$ be the number of star-shaped color classes of $c$. It is clear that an upper bound on the size of each star-shaped color class is $\left(\begin{array}{l}n-1 \\ k-1\end{array}\right)$. There are $n-\alpha-2 k+1$ many non-starshaped classes, and Lemma 7 bounds their size by $k^{2}\left(\begin{array}{l}n-2 \\ k-2\end{array}\right)$. This implies that

$$
\left(\begin{array}{l}
n-1 \\
k-1
\end{array}\right) \alpha+k^{2}\left(\begin{array}{l}
n-2 \\
k-2
\end{array}\right)(n-\alpha-2 k+1) \geq\left(\begin{array}{l}
n \\
k
\end{array}\right) .
$$

Assume for a contradiction that $\alpha<\frac{n}{k} \beta$. Since $n>\frac{k^{3}(k-\beta)}{1-\beta}, 0<\beta<1$, and $k \geq 2$, we have $n-1>k^{3}(k-1)>k^{2}(k-1)$. Therefore, $\left(\begin{array}{l}n-1 \\ k-1\end{array}\right)>k^{2}\left(\begin{array}{c}n-2 \\ k-2\end{array}\right)$, and if $\alpha$ is replaced by the larger value $\frac{n}{k} \beta$, the left hand side of (3) increases. Thus,

$$
\left(\begin{array}{l}
n-1 \\
k-1
\end{array}\right) \frac{n}{k} \beta+k^{2}\left(\begin{array}{l}
n-2 \\
k-2
\end{array}\right)\left(n-\frac{n}{k} \beta-2 k+1\right)>\left(\begin{array}{l}
n \\
k
\end{array}\right) \text {. }
$$

Since $\left(\begin{array}{l}n-1 \\ k-1\end{array}\right) \frac{n}{k}=\left(\begin{array}{l}n \\ k\end{array}\right)$ and $n-\frac{n}{k} \beta-2 k+1=\frac{k-\beta}{k} n-2 k+1$,

$$
k^{2}\left(\begin{array}{l}
n-2 \\
k-2
\end{array}\right)\left(\frac{k-\beta}{k} n-2 k+1\right)>(1-\beta)\left(\begin{array}{l}
n \\
k
\end{array}\right) \text {. }
$$


Expanding the binomial coefficients yields

$$
k^{3}(k-1)\left(\frac{k-\beta}{k} n-2 k+1\right)>(1-\beta) n(n-1) .
$$

We have $\frac{k-\beta}{k}(n-1)>\frac{k-\beta}{k} n-2 k+1$. Therefore,

$$
k^{3}(k-1) \frac{k-\beta}{k}(n-1)>(1-\beta) n(n-1) .
$$

Dividing by $n-1$ gives $k^{3}(k-\beta)>(1-\beta) n$, contradicting $n>\frac{k^{3}(k-\beta)}{1-\beta}$.

We now give our second proof of the Kneser-Lovász theorem.

Proof of Theorem 1, except for base cases. Fix $k>1$. By Lemma 9 with $\beta=1 / 2$, if $n>N(k, 1 / 2)$ and $c$ is an $(n-2 k+1)$ coloring of $\left(\begin{array}{l}n \\ k\end{array}\right)$, then $c$ has at least $n / 2 k$ many star-shaped color classes. We prove the Kneser-Lovász theorem by induction on $n$. The base cases are where $2 k \leq n \leq N(k, 1 / 2)$, and there are only finitely of these, so they can be exhaustively proven. For $n>N(k, 1 / 2)$, we structure the induction proof as an infinite descent. In other words, we show that if $c$ is an $(n-2 k+1)$-coloring of $\left(\begin{array}{l}n \\ k\end{array}\right)$, then there is some $c^{\prime}$ that is an $\left(\left(n-\frac{n}{2 k}\right)-2 k+1\right)$-coloring of $\left(\begin{array}{c}n-\frac{n}{2 k} \\ k\end{array}\right)$. For simplicity of notation, we assume $\frac{n}{2 k}$ is an integer. If this is not the case, we really mean to round up to the nearest integer $\left\lceil\frac{n}{2 k}\right\rceil$.

By permuting the color classes and the nodes, we can assume w.l.o.g. that the $\frac{n}{2 k}$ color classes $P_{\ell}$ for $\ell=n-\frac{n}{2 k}-$ $2 k+2, \ldots, n-2 k+1$ are star-shaped, and each such $P_{\ell}$ has a central node in $\{n-(n / 2 k)+1, \ldots, n\}$. That is, the last $\frac{n}{2 k}$ many color classes are star-shaped, and they all have a central node among the last $\frac{n}{2 k}$ nodes in [n]. We shall discard these $n / 2 k$ many star-shaped color classes, and the topmost $n / 2 k$ many nodes. This discards the central nodes of the discarded color classes, thereby removing all the vertices of the Kneser graph which are assigned discarded color classes. (It is possible that some star-shaped color classes share central nodes. We only need to be sure to discard at least one central node for each color classes, and thus, in this case, additional nodes can be discarded so that $n / 2 k$ are discarded in all.)

More formally, define $c^{\prime}$ to be the coloring of $\left(\begin{array}{c}n-n / 2 k \\ k\end{array}\right)$ which assigns the same colors as $c$. The map $c^{\prime}$ is a $\left(\frac{2 k-1}{2 k} n-\right.$ $2 k+1)$-coloring of $\left(\begin{array}{c}\frac{2 k-1}{2 k} n \\ k\end{array}\right)$, since $n-\frac{n}{2 k}=\frac{2 k-1}{2 k} n$. This completes the proof of the induction step.

When formalizing the above argument with quasi-polynomial size Frege proofs, it will be important to know how many iterations of the procedure are required to reach the base cases, so let us calculate this.

After $s$ iterations of this procedure, we have a $\left(\left(\frac{2 k-1}{2 k}\right)^{s} n-2 k+1\right)$-coloring of $\left(\begin{array}{c}\left(\frac{2 k-1}{2 k}\right)^{s} n \\ k\end{array}\right)$. We pick $s$ large enough so that $\left(\frac{2 k-1}{2 k}\right)^{s} n$ is less than $N(k, 1 / 2)$. In other words, since $k$ is constant,

$$
s=\log _{\frac{2 k}{2 k-1}}\left(\frac{n}{k^{3}(2 k-1)}\right)=O(\log n)
$$

will suffice, and only $O(\log n)$ many rounds of the procedure are required.

\subsection{Optimal colorings of Kneser graphs}

This section is a brief diversion motivated by the question of whether Lemma 9 about the number of non-starshaped colors is optimal.

It is well-known that $\left(\begin{array}{l}n \\ k\end{array}\right)$ has an $(n-2 k+2)$-coloring [25]. A simple construction of such a coloring, which we call $c_{1}$, is given here for completeness as follows. For $S \in\left(\begin{array}{l}n \\ k\end{array}\right)$, define $c_{1}(S)$ by:

(1) If $S \nsubseteq[2 k-1]$, let $c_{1}(S)=\max (S)-(2 k-2)$. Clearly $1<c_{1}(S) \leq n-2 k+2$.

(2) If $S \subseteq[2 k-1]$, let $c_{1}(S)=1$

We claim that $c_{1}$ defines a proper coloring. By construction, if $c_{1}(S)>1$, then $c_{1}(S)+(2 k-2) \in S$. Thus, if $c_{1}(S)=c_{1}\left(S^{\prime}\right)>1$, then $S \cap S^{\prime} \neq \emptyset$ and $S$ and $S^{\prime}$ are not joined by an edge in the Kneser graph. On the other hand, if $c_{1}(S)=1$, then $S$ contains $k$ elements from the set $[2 k-1]$. Any two such subsets have nonempty intersection, and therefore if $c_{1}(S)=c_{1}\left(S^{\prime}\right)=1$, then again $S \cap S^{\prime} \neq \emptyset$. Note that $c_{1}$ contains $n-2 k+1$ many star-shaped color classes, and only one non-starshaped color class.

In view of Lemma 9 , it is interesting to ask whether it is possible to give $(n-2 k+2)$-colorings which have fewer star-shaped color classes and more non-starshaped color classes. The next theorem gives the best construction we know.

Theorem 10. Let $k \geq 1$ and $n \geq 3 k-3$. There is an $(n-2 k+2)$ coloring $c_{k-1}$ of $\left(\begin{array}{l}n \\ k\end{array}\right)$ which has $k-1$ many non-starshaped color classes and only $n-3 k+3$ many star-shaped color classes. 
Proof. To construct $c_{k-1}$, partition the set $[n]$ into $n-2 k+2$ many subsets $T_{1}, \ldots, T_{n-2 k+2}$ as follows. For $i \leq n-3 k+3, T_{i}$ is chosen to be a singleton set, say $T_{i}=\{n-i+1\}$. The remaining $k-1$ many $T_{i}$ 's are subsets of size 3, say $T_{i}=\{j-2, j-1, j\}$ where $j=3(i-(n-3 k+3))$. Since $n=(n-3 k+3)+3(k-1)$, the sets $T_{i}$ partition [n], and each $T_{i}$ has cardinality either 1 or 3. For $S$ a subset of $n$ of cardinality $k$, define the color $c_{k-1}(S)$ to equal the least $i$ such that

$$
\left|S \cap T_{i}\right|>\frac{1}{2}\left|T_{i}\right|
$$

We claim there must exist such an $i$. If not, then $S$ contains no members of the singleton subsets $T_{i}$ and at most one member of each of the subsets $T_{i}$ of size three. But there are only $k-1$ many subsets of size three, contradicting $|S|=k$.

It is easy to check that if $c_{k-1}(S)=c_{k-1}\left(S^{\prime}\right)$ then $S \cap S^{\prime} \neq \emptyset$. Thus $c_{k-1}$ is a coloring. Furthermore, $c_{k-1}$ has $k-1$ many non-starshaped color classes and $n-3 k+3$ many star-shaped color classes.

Theorem 10 can be extended to show that when $2 k \leq n \leq 3 k-3$, there is a $n-2 k+2$ coloring with no star-shaped color class. The proof construction uses a similar idea, based on the fact that [ $n$ ] can be partitioned into $n-2 k+2 \leq k-1$ many subsets, each of odd cardinality $\geq 3$. We leave the details to the reader.

Question 11. Do there exist $(n-2 k+2)$-colorings of the $(n, k)$-Kneser graphs with more than $k-1$ many non-starshaped color classes?

\section{Formalization in propositional logic}

\subsection{Polynomial size extended Frege proofs}

We sketch the formalization of the argument in Section 3.1 as a polynomial size extended Frege proof, establishing Theorem 4. We concentrate on showing how to express concepts such as "star-shaped color class" with polynomial size propositional formulas. For expository reasons, we omit the straightforward details of how (extended) Frege proofs can prove properties of these concepts.

Fix values for $k$ and $n$ with $n>N(k)$. We describe an extended Frege proof of $\operatorname{Kneser}_{k}^{n}$. We have variables $p_{S, j}$ (recall Definition 3), collectively denoted $\vec{p}$. The proof assumes $\operatorname{Kneser}_{k}^{n}(\vec{p})$ is false, and proceeds by contradiction. The main step is to define new variables $\vec{p}^{\prime}$ with the extension rule and prove that $\operatorname{Kneser}_{k}^{n-1}\left(\vec{p}^{\prime}\right)$ fails. This will be repeated until reaching a Kneser graph over only $N(k)$ nodes.

For this, let $\operatorname{Star}(i, \ell)$ express that $i \in[n]$ is a central node of the color class $P_{\ell}$; namely,

$$
\operatorname{Star}(i, \ell):=\bigwedge_{S \in\left(\begin{array}{l}
n \\
k
\end{array}\right), i \notin S} \neg p_{S, \ell} .
$$

Note that $P_{\ell}$ may have more than one central node. Conversely, a node $i$ may be a central node for more than one color class.

We use $\operatorname{Star}(\ell):=\bigvee_{i} \operatorname{Star}(i, \ell)$ to express that $P_{\ell}$ is star-shaped.

The extended Frege proof defines an instance of the Kneser-Lovász principle Kneser $_{k}^{n-1}$ by discarding one node and one color. The first star-shaped color class $P_{\ell}$ is discarded; accordingly, we let

$$
\operatorname{DiscardColor}(\ell):=\operatorname{Star}(\ell) \wedge \bigwedge_{\ell^{\prime}<\ell} \neg \operatorname{Star}\left(\ell^{\prime}\right) .
$$

The node to be discarded is the least central node of the discarded $P_{\ell}$ :

$$
\operatorname{DiscardNode}(i):=\bigvee_{\ell}\left[\operatorname{DiscardColor}(\ell) \wedge \operatorname{Star}(i, \ell) \wedge \bigwedge_{i^{\prime}<i} \neg \operatorname{Star}\left(i^{\prime}, \ell\right)\right]
$$

After discarding the node $i$ and the color $\ell$, the remaining nodes and colors are renumbered to the ranges [ $n-1]$ and $[n-2 k]$, respectively. In particular, the "new" color $j$ (in the instance of $\operatorname{Kneser}_{k}^{n-1}$ ) corresponds to the "old" color $j^{-\ell}$ (in the instance of $\operatorname{Kneser}_{k}^{n}$ ) where

$$
j^{-\ell}= \begin{cases}j & \text { if } j<\ell \\ j+1 & \text { if } j \geq \ell\end{cases}
$$

And, if $S=\left\{i_{1}, \ldots, i_{k}\right\} \in\left(\begin{array}{c}n-1 \\ k\end{array}\right)$ is a "new" vertex (for the Kneser ${ }_{k}^{n-1}$ instance), then it corresponds to the "old" vertex $S^{-i} \in\left(\begin{array}{l}n \\ k\end{array}\right)$ (for the instance of Kneser $_{k}^{n}$ ), where $S^{-i}=\left\{i_{1}^{\prime}, i_{2}^{\prime}, \ldots, i_{k}^{\prime}\right\}$ with 


$$
i_{t}^{\prime}= \begin{cases}i_{t} & \text { if } i_{t}<i \\ i_{t}+1 & \text { if } i_{t} \geq i .\end{cases}
$$

For each $S \in\left(\begin{array}{c}n-1 \\ k\end{array}\right)$ and $j \in[n-2 k]$, the extended Frege proof uses the extension rule to introduce a new variable $p_{S, j}^{\prime}$ defined as follows

$$
p_{S, j}^{\prime} \equiv \bigvee_{i, \ell}\left(\operatorname{DiscardNode}(i) \wedge \operatorname{DiscardColor}(\ell) \wedge p_{S^{-i}, j^{-\ell}}\right)
$$

As seen in the definition by extension, $p_{S, j}^{\prime}$ is defined by cases, one for each possible pair $i, \ell$ of nodes and colors such that the node $i$ is the least central node of the $P_{\ell}$ color class, where $P_{\ell}$ is the first star-shaped color class. The extended Frege proof then shows that $\neg \operatorname{Kneser}_{k}^{n}(\vec{p})$ implies $\neg \operatorname{Kneser}_{k}^{n-1}\left(\vec{p}^{\prime}\right)$, i.e., that if the variables $p_{S, j}$ define a coloring, then the variables $p_{S, j}^{\prime}$ also define a coloring. The first step for the extended Frege proof is to show that there is at least one star-shaped color class, and then there is a unique $\ell$ such that DiscardColor $(\ell)$ holds. In fact, we claim there are polynomial size Frege proofs of

$$
\bigvee_{\ell} \operatorname{DiscardColor}(\ell)
$$

and

$$
\bigwedge_{\ell_{1}<\ell_{2}}\left(\neg \operatorname{DiscardColor}\left(\ell_{1}\right) \vee \neg \operatorname{DiscardColor}\left(\ell_{2}\right)\right) .
$$

The Frege proof of (4) and (5) starts by proving $\bigvee_{\ell} \operatorname{Star}(\ell)$. This is done essentially via a proof by contradiction: First, under the hypothesis that $\neg \bigvee_{\ell} \operatorname{Star}(\ell)$, the Frege proof uses the argument of Lemma 7 to show, for each color class $P_{\ell}$, that there is a surjective map $\pi_{\ell}$ from $\left[k^{2}\left(\begin{array}{l}n-2 \\ k-2\end{array}\right)\right]$ onto $P_{\ell}$. Fixing a particular value for $\ell$, the Frege proof defines $\pi_{\ell}$ as follows: It chooses a set $S_{0}$ in $P_{\ell}$, say the lexicographically first set $S_{0}$ in $P_{\ell}$. There are $\leq\left(\begin{array}{l}n \\ k\end{array}\right)$ many possible choices for this $S_{0}$; and the Frege proof splits into cases based on $S_{0}$. Then, letting $S_{0}=\left\{a_{1}, \ldots, a_{k}\right\}$ in increasing order, the Frege proof proves, for each $i$ the existence of a lexicographically first set $S_{i}$ in $P_{\ell}$ with $a_{i} \notin S_{i}$ (using the assumption that $P_{\ell}$ is not star-shaped). The Frege proof further splits into polynomially many cases for all possible choices of $S_{1}, \ldots, S_{k}$. Let $a_{i, i^{\prime}}$ be the $i^{\prime}$-th member of $S_{i}$. For each $j \neq j^{\prime} \in[n]$, there is a natural bijection $\pi_{j, j^{\prime}}$ from $\left(\begin{array}{c}n-2 \\ k-2\end{array}\right)$ to the $(k-2)$-subsets of $[n] \backslash\left\{j, j^{\prime}\right\}$. For $i, i^{\prime} \in[k]$ and $p \in\left(\begin{array}{l}n-2 \\ k-2\end{array}\right)$, the surjection $\pi_{\ell}$ can be defined by $\pi\left(i, i^{\prime}, p\right)=\left\{a_{i}, a_{i, i^{\prime}}, \pi_{a_{i}, a_{i, i^{\prime}}}(p)\right\}$ if this a well-defined member of $P_{\ell}$, and $\pi\left(i, i^{\prime}, p\right)=S_{0}$ otherwise. The intersection properties of $P_{\ell}$, as in the proof of Lemma 7 , show immediately that $\pi_{\ell}$ is surjective.

Using the formalization of "counting" in Frege proofs [9], the surjectivity of $\pi_{\ell}$ implies that $\left|P_{\ell}\right| \leq k^{2}\left(\begin{array}{c}n-2 \\ k-2\end{array}\right)$. But since $(n-2 k+1) k^{2}\left(\begin{array}{l}n-2 \\ k-2\end{array}\right)<\left(\begin{array}{l}n \\ k\end{array}\right)$, this contradicts the fact that every vertex is in a color class. The fact that $(n-2 k+1) k^{2}\left(\begin{array}{l}n-2 \\ k-2\end{array}\right)<\left(\begin{array}{l}n \\ k\end{array}\right)$ is proved by just computing the values of both sides of the inequality. Indeed, $k$ is fixed, so there are only $\left(\begin{array}{l}n \\ k\end{array}\right)<n^{k}$ many vertices, and we are only counting polynomially many vertices.

Once $\bigvee_{\ell} \operatorname{Star}(\ell)$ has been proved with a polynomial size proof (under the hypothesis that $\neg \operatorname{Kneser}_{k}^{n}(\vec{p})$ ), the formulas (4) and (5) follow easily. Likewise, there are polynomial size Frege proofs that there is a unique value $i \in[n-2 k+1]$ which satisfies DiscardNode $(i)$.

For fixed values of $\ell$ and $i$, a polynomial size Frege proof now establishes

$$
\operatorname{DiscardColor}(\ell) \wedge \operatorname{DiscardNode}(i) \wedge \operatorname{Kneser}_{k}^{n-1}\left(\vec{p}^{\prime}\right) \rightarrow \operatorname{Kneser}_{k}^{n}(\vec{p})
$$

This Frege proof argues as follows, assuming DiscardColor $(\ell)$ and DiscardNode $(i)$ and $\operatorname{Kneser}_{k}^{n-1}\left(\vec{p}^{\prime}\right)$. Since $\operatorname{Kneser}_{k}^{n-1}\left(\vec{p}^{\prime}\right)$ is true, either (a) its hypothesis is false and we have $\bigwedge_{j=1}^{n-2 k} \neg p_{S, j}^{\prime}$ for some $S \in\left(\begin{array}{l}n \\ k\end{array}\right)$ or (b) its conclusion is true and there are $S, T \in\left(\begin{array}{l}n \\ k\end{array}\right)$ and $j$ such that $S \cap T=\emptyset$ and $p_{S, j}^{\prime}$ and $p_{T, j}^{\prime}$. If (a) holds then $\neg p_{S^{-i}, j^{-\ell}}$ for all $j \in[n-2 k]$ and this together with

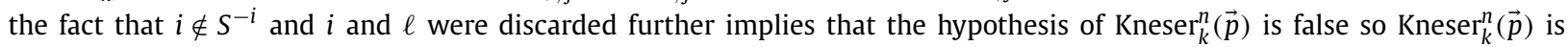
true. Likewise, if (b) holds, then using $S^{-i}$ and $T^{-i}$ and $j^{-\ell}$ shows that the conclusion of Kneser $k_{k}^{n}$ is true.

Putting all these arguments together gives the desired Frege proof of

$$
\neg \operatorname{Kneser}_{k}^{n}(\vec{p}) \rightarrow \neg \operatorname{Kneser}_{k}^{n-1}\left(\vec{p}^{\prime}\right) .
$$

The extended Frege proof iterates this process of removing one node and one color until it is shown that there is a coloring of $\left(\begin{array}{c}N(k) \\ k\end{array}\right)$. This is then refuted by exhaustively considering all colorings of Kneser graphs on $\leq N(k)$ nodes. 


\subsection{Quasi-polynomial size Frege proofs}

This section discusses some of the details of the formalization of the argument in Section 3.2 as quasi-polynomial size Frege proofs, establishing Theorem 5. First we will form an extended Frege proof, then modify it to become a Frege proof. As before, the proof starts with the assumption that $\operatorname{Kneser}_{k}^{n}(\vec{p})$ is false. As we describe next, the extended Frege proof then introduces variables $\vec{p}^{\prime}$ by extension so that $\operatorname{Kneser}_{k}^{n-n / 2 k}\left(\vec{p}^{\prime}\right)$ is false. This process will be repeated $O(\log n)$ times. The final Frege proof is obtained by unwinding the definitions by extension.

For a set $X$ of formulas and $t>0$, we now use the notation " $|X| \leq t$ " to denote a formula that is true when the number of true formulas in $X$ is less than or equal to $t$. As already discussed, " $|X| \leq t$ " can be expressed by a formula of size polynomially bounded by the total size of the formulas in $X$, using the construction in [9]. " $|X|=t$ " is defined similarly.

The formulas $\operatorname{Star}(i, \ell)$ and $\operatorname{Star}(\ell)$ are the same as in Section 4.1. A color $\ell$ is now discarded if it is among the least $n / 2 k$ star-shaped color classes.

$$
\operatorname{DiscardColor}(\ell):=\operatorname{Star}(\ell) \wedge\left(\left|\left\{\operatorname{Star}\left(\ell^{\prime}\right): \ell^{\prime} \leq \ell\right\}\right| \leq n / 2 k\right)
$$

The discarded nodes are the least central nodes of the discarded color classes.

$$
\operatorname{DiscardNode}(i):=\bigvee_{\ell}\left[\operatorname{DiscardColor}(\ell) \wedge \operatorname{Star}(i, \ell) \wedge \bigwedge_{i^{\prime}<i} \neg \operatorname{Star}\left(i^{\prime}, \ell\right)\right] \text {. }
$$

DiscardNode $(i)$ will hold for at most $n / 2 k$ many nodes $i$, since there are only $n / 2 k$ many discarded colors. We could modify the definition of DiscardNode to discard exactly $n / 2 k$ many nodes; however, this is not strictly necessary, as the only use of DiscardNode is to define the predicate RenumNode $\left(i^{\prime}, i\right)$ below, and that definition effectively discards exactly $n / 2 k$ many nodes even if DiscardNode $(i)$ picks out fewer than $n / 2 k$ many nodes to be discarded.

The remaining, non-discarded colors and nodes are renumbered to form an instance of $\operatorname{Kneser}_{k}^{n-n / 2 k}$. For this, the formula RenumNode $\left(i^{\prime}, i\right)$ is true when the node $i^{\prime}$ is the $i$-th node that is not discarded; similarly $\operatorname{RenumColor}\left(j^{\prime}, j\right)$ is true when the color $j^{\prime}$ is the $j$-th color that is not discarded.

$$
\begin{aligned}
& \operatorname{RenumNode}\left(i^{\prime}, i\right):=\left(\left|\left\{\neg \operatorname{DiscardNode}\left(i^{\prime \prime}\right): i^{\prime \prime} \leq i^{\prime}\right\}\right|=i\right) \wedge \neg \operatorname{DiscardNode}\left(i^{\prime}\right) \\
& \operatorname{RenumColor}\left(j^{\prime}, j\right):=\left(\left|\left\{\neg \operatorname{DiscardColor}\left(j^{\prime \prime}\right): j^{\prime \prime} \leq j^{\prime}\right\}\right|=j\right) \wedge \neg \operatorname{DiscardColor}\left(j^{\prime}\right)
\end{aligned}
$$

The predicate RenumNode $\left(i^{\prime}, i\right)$ defines a bijection between the sets $[n-n / 2 k]$ and the non-discarded nodes of $[n]$. Likewise, the predicate RenumColor $\left(j^{\prime}, j\right)$ defines a bijection between $[(n-n / 2 k)-2 k+1]$ and the non-discarded colors.

For each $S=\left\{i_{1}, \ldots, i_{k}\right\} \in\left(\begin{array}{c}n-n / 2 k \\ k\end{array}\right)$ and $j \in[(n-n / 2 k)-2 k+1]$, we define by extension

$$
p_{S, j}^{\prime} \equiv \bigvee_{i_{1}^{\prime}, \ldots i_{k}^{\prime}, j^{\prime}}\left(\bigwedge_{t=1}^{k}\left(\operatorname{RenumNode}\left(i_{t}^{\prime}, i_{t}\right)\right) \wedge \operatorname{RenumColor}\left(j^{\prime}, j\right) \wedge p_{\left\{i_{1}^{\prime}, \ldots, i_{k}^{\prime}\right\}, j^{\prime}}\right)
$$

The Frege proof then argues that if the variables $p_{S, j}$ define a coloring, then the variables $p_{S, j}^{\prime}$ define a coloring, i.e., that $\neg \operatorname{Kneser}_{k}^{n}(\vec{p}) \rightarrow \neg \operatorname{Kneser}_{k}^{n-n / 2 k}\left(\vec{p}^{\prime}\right)$. The first step for this is proving that there are at least $n / 2 k$ star-shaped color classes by formalizing the proofs of Lemmas 7 and 9. Those proofs were "counting" arguments: they involved counting the number of members of $\left(\begin{array}{l}n \\ k\end{array}\right)$ that are contained in the color classes $P_{\ell}$. As already mentioned, the proof of Lemma 7 can be formalized with polynomial size Frege proofs proving that, if $P_{\ell}$ is a non-starshaped color class, there is a surjective map from $\left[k^{2}\left(\begin{array}{l}n-2 \\ k-2\end{array}\right)\right]$ onto $P_{\ell}$ and from this concluding that $\left|P_{\ell}\right| \leq k^{2}\left(\begin{array}{l}n-2 \\ k-2\end{array}\right)$. Similarly, and even easier, there are polynomial size Frege proofs of the fact that if $P_{\ell}$ is star-shaped, then there is a surjective map from $\left(\begin{array}{c}n-1 \\ k-1\end{array}\right)$ onto $P_{\ell}$, from whence $\left|P_{\ell}\right| \leq$ $\left(\begin{array}{l}n-1 \\ k-1\end{array}\right)$. The Frege proof then splits into polynomially many cases depending on the number $\alpha$ of star-shaped color classes. For each $\alpha$, the inequality (3) must hold by the upper bounds on the $\left|P_{\ell}\right|$ 's. However, for any fixed value of $\alpha<\frac{n}{k} \beta=\frac{n}{2 k}$, directly substituting the (fixed) values of $n, k, \alpha$ into (3) shows that it is false. ${ }^{6}$ It follows that $\alpha \geq \frac{n}{2 k}$; that is, there are $\geq \frac{n}{2 k}$ many star-shaped colors. From this, it follows, again with a polynomial size Frege proof, that RenumNode $\left(i^{\prime}, i\right)$ and RenumColor $\left(j^{\prime}, j\right)$ define bijections.

After that, it is straightforward to prove that, for each $S \in\left(\begin{array}{c}n-n / 2 k \\ k\end{array}\right)$ and $j \in[(n-n / 2 k)-2 k+1]$, the variable $p_{S, j}^{\prime}$ is well-defined. In addition, a polynomial size Frege proof can prove that if $\operatorname{Kneser}_{k}^{n}(\vec{p})$ is false, then $\operatorname{Kneser}_{k}^{n-n / 2 k}\left(\vec{p}^{\prime}\right)$ is false.

This is iterated $O(\log n)$ times until fewer than $N(k, 1 / 2)$ nodes remain. The proof concludes with a hard-coded proof that there are no such colorings of the finitely many small Kneser graphs.

To form the quasi-polynomial size Frege proof, we unwind the definitions by extension. Each definition by extension was polynomial size; they are nested to a depth of $O(\log n)$. So the resulting Frege proof is quasi-polynomial size.

\footnotetext{
6 We know that (3) is false by the argument given in the proof of Lemma 9; therefore a Frege proof can use direct calculation to verify this for the needed values of $n, k, \alpha$. Alternatively, a polynomial size Frege proof can carry out all the steps of the argument used earlier to establish ( 3 ); however, this is not necessary, and does not seem to add anything apart from possibly a bit more uniformity.
} 


\section{The octahedral Tucker lemma and truncated Tucker lemmas}

A natural question arising from the previous sections is the possibility of giving short uniform Frege proofs of the KneserLovász theorem for fixed $k$, namely, proofs that avoid handling finitely many base cases separately. A possible approach to this problem is formalizing the proof of Matoušek [26] in the Frege system. A significant obstacle in carrying this out is that Matoušek's proof goes through the octahedral Tucker lemma, and, as will be discussed below, naïve propositional translations of the octahedral Tucker lemma are of exponential size. To overcome this, we describe two miniaturizations of the octahedral Tucker lemma, called the truncated Tucker lemmas; these have polynomial size propositional translations, and are strong enough to imply the Kneser-Lovász theorem with polynomial size, constant depth Frege proofs.

Our definitions and proofs below borrow techniques and notation from Matoušek [26].

Definition 12. Let $n \geq 1$. The octahedral ball $\mathcal{B}^{n}$ is:

$$
\mathcal{B}^{n}:=\{(A, B): A, B \subseteq[n] \text { and } A \cap B=\emptyset\} .
$$

Definition 13. Let $n>1$. A mapping $\lambda: \mathcal{B}^{n} \rightarrow\{1, \pm 2, \ldots, \pm n\}$ is antipodal if $\lambda(\emptyset, \emptyset)=1$, and for all other pairs $(A, B) \in \mathcal{B}^{n}$, $\lambda(A, B)=-\lambda(B, A)$.

Note that -1 is not in the range of $\lambda$, and $(\emptyset, \emptyset)$ is the only member of $\mathcal{B}^{n}$ that is mapped to 1 by $\lambda$.

Definition 14. Two pairs $\left(A_{1}, B_{1}\right)$ and $\left(A_{2}, B_{2}\right)$ in $\mathcal{B}^{n}$ are complementary with respect to $\lambda$ if $A_{1} \subseteq A_{2}$, B $\subseteq B_{2}$ and $\lambda\left(A_{1}, B_{1}\right)=-\lambda\left(A_{2}, B_{2}\right)$.

Theorem 15 (Octahedral Tucker lemma). If $\lambda: \mathcal{B}^{n} \rightarrow\{1, \pm 2, \ldots, \pm n\}$ is antipodal, then there are two elements in $\mathcal{B}^{n}$ that are complementary.

For a proof of Theorem 15, see [26].

Definition 16. Let $1 \leq k \leq n$. The truncated octahedral ball $\mathcal{B}_{\leq k}^{n}$ is:

$$
\mathcal{B}_{\leq k}^{n}:=\left\{(A, B) \in \mathcal{B}^{n}:|A| \leq k,|B| \leq k\right\}
$$

We write $\left(\begin{array}{c}n \\ \leq k\end{array}\right)$ for $\{A \subseteq[n]:|A| \leq k\}$.

The octahedral Tucker lemma used the subset relation $\subseteq$ on $[n]$ to define "complementary". The truncated Tucker lemma uses an analogous partial order $\preceq$ to define " $k$-complementary". For $A \subseteq[n]$, let $A_{\leq k}$ denote the least $k$ elements of $A$. By convention, if $|A|<k$, then $A_{\leq k}=A$.

Definition 17. Let $\preceq$ be the partial order on sets in $\left(\begin{array}{c}n \\ \leq k\end{array}\right)$ defined by $A \preceq B$ iff $(A \cup B)_{\leq k}=B$.

Note that if $|B|=k$, then $A \preceq B$ is equivalent to the statement that every member of $A \backslash B$ is $\geq \max (B)$. On the other hand, if $|B|<k$, then $A \preceq B$ is equivalent to $A \subseteq B$. When $k=n, \mathcal{B}^{n}=\mathcal{B}_{\leq k}^{n}$, and the $\preceq$ relation is identical to the subset relation.

Lemma 18. The relation $\preceq$ is a partial order with $\emptyset$ its least element.

Proof. It is clearly reflexive. For anti-symmetry, $A_{1} \preceq A_{2}$ and $A_{2} \preceq A_{1}$ imply that $A_{1}=\left(A_{1} \cup A_{2}\right)_{\leq k}=\left(A_{2} \cup A_{1}\right)_{\leq k}=A_{2}$. For transitivity, suppose $A_{1} \preceq A_{2}$ and $A_{2} \preceq A_{3}$. Then $\left(A_{1} \cup A_{2}\right)_{\leq k}=A_{2}$ and $\left(A_{2} \cup A_{3}\right)_{\leq k}=A_{3}$. This implies

$$
A_{3}=\left(A_{2} \cup A_{3}\right)_{\leq k}=\left(\left(A_{1} \cup A_{2}\right)_{\leq k} \cup A_{3}\right)_{\leq k}=\left(A_{1} \cup\left(A_{2} \cup A_{3}\right)_{\leq k}\right)_{\leq k}=\left(A_{1} \cup A_{3}\right)_{\leq k}
$$

Therefore $A_{1} \preceq A_{3}$. That $\emptyset$ is the least element is clear from the definition.

Definition 19. For $\left(A_{1}, B_{1}\right)$ and $\left(A_{2}, B_{2}\right)$ in $\mathcal{B}_{\leq k}^{n}$, write $\left(A_{1}, B_{1}\right) \preceq\left(A_{2}, B_{2}\right)$ when $A_{1} \preceq A_{2}, B_{1} \preceq B_{2}$, and $A_{i} \cap B_{j}=\emptyset$ for $i, j \in\{1,2\}$. The pairs $\left(A_{1}, B_{1}\right)$ and $\left(A_{2}, B_{2}\right)$ are $k$-complementary with respect to an antipodal map $\lambda$ on $\mathcal{B}_{\leq k}^{n}$ if $\left(A_{1}, B_{1}\right) \preceq$ $\left(A_{2}, B_{2}\right)$ and $\lambda\left(A_{1}, B_{1}\right)=-\lambda\left(A_{2}, B_{2}\right)$.

We are ready to state the version of the truncated Tucker lemma for $\mathcal{B}_{\leq k}^{n}$. 
Theorem 20 (Truncated Tucker lemma on $\mathcal{B}_{\leq k}^{n}$ ). Suppose $n \geq k \geq 1$. If $\lambda: \mathcal{B}_{\leq k}^{n} \rightarrow\{1, \pm 2 \ldots, \pm n\}$ is antipodal, then there are two elements in $\mathcal{B}_{\leq k}^{n}$ that are $k$-complementary.

When $k=n$, this is equivalent to the octahedral Tucker lemma. The truncated Tucker lemma on $\mathcal{B}_{\leq k}^{n}$ follows from the octahedral Tucker lemma:

Proof of Theorem 20 from Theorem 15. We argue by contradiction. Suppose $\lambda: \mathcal{B}_{\leq k}^{n} \rightarrow\{1, \pm 2, \ldots, \pm n\}$ is antipodal. We define $\lambda^{\prime}: \mathcal{B}^{n} \rightarrow\{1, \pm 2, \ldots, \pm n\}$. For $(A, B) \in \mathcal{B}^{n}$, define $\lambda^{\prime}(A, B)=\lambda\left(A_{\leq k}, B_{\leq k}\right)$. The map $\lambda^{\prime}$ is clearly antipodal, so by Theorem 15 , there are $(A, B),(C, D)$ in $\mathcal{B}^{n}$ that are complementary with respect to $\lambda^{\prime}$. We claim that $\left(A_{\leq k}, B_{\leq k}\right)$ and $\left(C_{\leq k}, D_{\leq k}\right)$ are $k$-complementary with respect to $\lambda$. By definition of $\lambda^{\prime}, \lambda\left(A_{\leq k}, B_{\leq k}\right)=-\lambda\left(C_{\leq k}, D_{\leq k}\right)$, so it remains to show that $\left(A_{\leq k}, B_{\leq k}\right) \preceq\left(C_{\leq k}, D_{\leq k}\right)$. Since $C \cap D=\emptyset$ and $A \subseteq C$ and $B \subseteq D$, it follows that

$$
C_{\leq k} \cap D_{\leq k}=A_{\leq k} \cap D_{\leq k}=A_{\leq k} \cap B_{\leq k}=B_{\leq k} \cap C_{\leq k}=\emptyset .
$$

Moreover, $A \subseteq C$ implies that $A_{\leq k} \preceq C_{\leq k}$. This is because

$$
\left(A_{\leq k} \cup C_{\leq k}\right)_{\leq k}=(A \cup C)_{\leq k}=C_{\leq k} .
$$

The same argument shows that $B_{\leq k} \preceq D_{\leq k}$.

Definition 21. Let $1<2 k \leq n$. The truncated octahedral ball $\mathcal{B}_{k}^{n}$ is:

$$
\mathcal{B}_{k}^{n}:=\left\{(A, B): A, B \in\left(\begin{array}{l}
n \\
k
\end{array}\right) \cup\{\emptyset\}, A \cap B=\emptyset \text {, and }(A, B) \neq(\emptyset, \emptyset)\right\} .
$$

The fact that $(\varnothing, \emptyset)$ is excluded from $\mathcal{B}_{k}^{n}$ is only a technical convenience. Corresponding to this, the value " 1 " will now be omitted from the range of $\lambda$. We say that $\lambda: \mathcal{B}_{k}^{n} \rightarrow\{ \pm 2 k \ldots, \pm n\}$ is antipodal provided that $\lambda(A, B)=-\lambda(B, A)$ for all $(A, B) \in \mathcal{B}_{k}^{n}$.

Theorem 22 (Truncated Tucker lemma on $\mathcal{B}_{k}^{n}$ ). Suppose $n \geq 2 k>1$. If $\lambda: \mathcal{B}_{k}^{n} \rightarrow\{ \pm 2 k \ldots, \pm n\}$ is antipodal, then there are two elements in $\mathcal{B}_{k}^{n}$ that are $k$-complementary.

Proof of Theorem 22 from Theorem 20. Suppose that $\lambda: \mathcal{B}_{k}^{n} \rightarrow\{ \pm 2 k, \ldots, \pm n\}$ is antipodal; we must show it has $k$-complementary pairs. We extend $\lambda$ to an antipodal $\lambda^{\prime}: \mathcal{B}_{\leq k}^{n} \rightarrow\{1, \pm 2, \cdots \pm n\}$. Let “ $\leq$ ” be any total order on $\left(\begin{array}{c}n \\ \leq k\end{array}\right)$ that extends $\preceq$. Let $(A, B) \in \mathcal{B}_{\leq k}^{n}$. The value of $\lambda^{\prime}(A, B)$ is defined by cases:

Case 1: If $|A|<k$ and $|B|<k$, then define

$$
\lambda^{\prime}(A, B)= \begin{cases}1+|A|+|B| & \text { if } A \leq B \\ -(1+|A|+|B|) & \text { if } B<A .\end{cases}
$$

Case 2: If $\max \{|A|,|B|\}=k$ and $\min \{|A|,|B|\}<k$, then define

$$
\lambda^{\prime}(A, B)= \begin{cases}\lambda(A, \emptyset) & \text { if }|B|<k \\ \lambda(\emptyset, B) & \text { if }|A|<k\end{cases}
$$

Case 3: If $|A|=|B|=k$, then define $\lambda^{\prime}(A, B)=\lambda(A, B)$.

The map $\lambda^{\prime}$ is clearly antipodal; hence Theorem 20 implies there exist $\left(A_{1}, B_{1}\right) \preceq\left(A_{2}, B_{2}\right)$ which are $k$-complementary with respect to $\lambda^{\prime}$, so we have $\lambda^{\prime}\left(A_{1}, B_{1}\right)=-\lambda^{\prime}\left(A_{2}, B_{2}\right)$. We prove this gives rise to $k$-complementary pairs for $\lambda$. The argument splits into cases depending on how $\lambda^{\prime}$ assigns values to $\left(A_{1}, B_{1}\right)$ and $\left(A_{2}, B_{2}\right)$.

Suppose that one of $\lambda^{\prime}\left(A_{1}, B_{1}\right)$ or $\lambda^{\prime}\left(A_{2}, B_{2}\right)$ is assigned by case 1 . Since case 1 only assigns values from $\{1, \pm 2, \ldots$, $\pm(2 k-1)\}$, and cases 2 and 3 only assign values from $\{ \pm 2 k, \ldots, \pm n\}$, this implies that both $\lambda^{\prime}\left(A_{1}, B_{1}\right)$ and $\lambda^{\prime}\left(A_{2}, B_{2}\right)$ are assigned by case 1 . Also, $A_{1} \preceq A_{2}$ and $B_{1} \preceq B_{2}$ where at least one of these precedences is proper. For sets of cardinality less than $k$, the $\preceq$ relation is equivalent to the subset relation; therefore, we have $A_{1} \subseteq A_{2}$ and $B_{1} \subseteq B_{2}$ where again at least one of the inclusions is proper. Thus $1+\left|A_{1}\right|+\left|B_{1}\right|<1+\left|A_{2}\right|+\left|B_{2}\right|$, so $\lambda^{\prime}\left(A_{1}, B_{1}\right)$ and $\lambda^{\prime}\left(A_{2}, B_{2}\right)$ differ in absolute value. This contradicts the fact that $\left(A_{1}, B_{1}\right)$ and $\left(A_{2}, B_{2}\right)$ are $k$-complementary w.r.t. $\lambda^{\prime}$. Thus it is impossible that either $\lambda^{\prime}\left(A_{1}, B_{1}\right)$ or $\lambda^{\prime}\left(A_{2}, B_{2}\right)$ is assigned by case 1 .

Suppose $\lambda^{\prime}\left(A_{1}, B_{1}\right)$ and $\lambda^{\prime}\left(A_{2}, B_{2}\right)$ are both assigned by case 2 . Without loss of generality $\left|B_{1}\right|<k$, which implies $\left|A_{1}\right|=$ $\left|A_{2}\right|=k$ and $\left|B_{2}\right|<k$. This implies that $\lambda\left(A_{1}, \emptyset\right)=-\lambda\left(A_{2}, \emptyset\right)$. But $\left(A_{1}, \emptyset\right) \preceq\left(A_{2}, \emptyset\right)$, so these form a $k$-complementary pair for $\lambda$. 
Suppose $\lambda^{\prime}\left(A_{1}, B_{1}\right)$ is assigned by case 2 and $\lambda^{\prime}\left(A_{2}, B_{2}\right)$ is assigned by case 3 . Without loss of generality $\left|B_{1}\right|<k$. This implies that $\lambda\left(A_{1}, \emptyset\right)=-\lambda\left(A_{2}, B_{2}\right)$. But $\left(A_{1}, \emptyset\right) \preceq\left(A_{2}, B_{2}\right)$, so these form a $k$-complementary pair for $\lambda$.

Suppose $\lambda^{\prime}\left(A_{1}, B_{1}\right)$ and $\lambda^{\prime}\left(A_{2}, B_{2}\right)$ are both assigned by case 3 . In this case, $\lambda\left(A_{1}, B_{1}\right)=-\lambda\left(A_{2}, B_{2}\right)$, so these form a $k$-complementary pair for $\lambda$.

Suppose $\lambda^{\prime}\left(A_{1}, B_{1}\right)$ is assigned by case 3 and $\lambda^{\prime}\left(A_{2}, B_{2}\right)$ is assigned by case 2 . This is impossible because $\left|A_{1}\right|=\left|B_{1}\right|=k$, and $A_{1} \preceq A_{2}, B_{1} \preceq B_{2}$, so $\left|B_{1}\right|=\left|B_{2}\right|=k$.

For fixed parameter $k$, the two truncated Tucker lemmas have polynomial size propositional translations. We will only describe the translation of the truncated Tucker lemma on $\mathcal{B}_{k}^{n}$. A similar translation works for the truncated Tucker lemma on $\mathcal{B}_{<k}^{n}$. For each $(A, B) \in \mathcal{B}_{k}^{n}$, and for each $i \in\{ \pm 2 k, \ldots, \pm n\}$, let $p_{A, B, i}$ be a propositional variable with the intended meaning that $p_{A, B, i}$ is true when $\lambda(A, B)=i$. The following formula $\operatorname{Ant}(\vec{p})$ states that the map is total and antipodal:

$$
\bigwedge_{(A, B) \in \mathcal{B}_{k}^{n}} \bigvee_{i \in\{ \pm 2 k, \ldots, \pm n\}}\left(p_{A, B, i} \wedge p_{B, A,-i}\right)
$$

The following formula $\operatorname{Comp}(\vec{p})$ states that there exists two elements in $\mathcal{B}_{k}^{n}$ that are $k$-complementary:

$$
\bigvee_{\substack{\left(A_{1}, B_{1}\right),\left(A_{2}, B_{2}\right) \in \mathcal{B}_{k}^{n},\left(A_{1}, B_{1}\right) \leq\left(A_{2}, B_{2}\right) \\ i \in\{ \pm 2 k, \ldots, \pm n\}}}\left(p_{A_{1}, B_{1}, i} \wedge p_{A_{2}, B_{2},-i}\right) .
$$

The truncated Tucker tautology $\operatorname{Tucker}_{k}^{n}$ is defined to be $\operatorname{Ant}(\vec{p}) \rightarrow \operatorname{Comp}(\vec{p})$. (We could add an additional hypothesis, that for each $A, B$ there is at most one $i$ such that $p_{A, B, i}$, but this is not needed for the truncated Tucker tautologies to be valid.) There are $<n^{2 k}$ members $(A, B)$ in $\mathcal{B}_{k}^{n}$. Hence, for fixed $k$, there are only polynomially many variables $p_{A, B, i}$, and the truncated Tucker tautologies have size polynomially bounded by $n$. On the other hand, the propositional translation of the octahedral Tucker lemma requires an exponential number of propositional variables in $n$, since the cardinality of $\mathcal{B}^{n}$ is exponential in $n$.

We claim that the proof of Theorem 22 from Theorem 20 can be translated into polynomial size Frege proofs. Namely, if all instances of the propositional translations of the truncated Tucker lemma on $\mathcal{B}_{<k}^{n}$ are given as hypotheses, then there are polynomial size Frege proofs of the propositional translations of the truncated Tucker lemma on $\mathcal{B}_{k}^{n}$. These Frege proofs have propositional variables $p_{A, B, \alpha}$ indicating that $\lambda(A, B)=\alpha$. They start by assuming that $\lambda$ as encoded by these propositional variables does not satisfy (the propositional translation of) the statement of Theorem 22. The function $\lambda^{\prime}$ was defined in terms of $\lambda$ by a simple set of cases; hence there are polynomial-size formulas $\varphi_{A, B, \alpha}$ defining the condition $\lambda^{\prime}(A, B)=\alpha$. Invoking the instance of Theorem 20 with the formulas $\varphi$, there must be a $k$-complementary pair $(A, B)$ for $\lambda^{\prime}$ as encoded by the formulas $\varphi_{A, B, \alpha}$. A simple case analysis using the proof of Theorem 22 now gives a complementary pair for $\lambda$ as encoded by the variables $p_{A, B, \alpha}$. This suffices to give polynomial size Frege proofs of translations of Theorem 22 from Theorem 20.

In Section 5.1 will prove a converse to this: There are polynomial size Frege proofs of the propositional translations of the truncated Tucker lemma on $\mathcal{B}_{\leq k}^{n}$ given instances of the propositional translations of the truncated Tucker lemma on $\mathcal{B}_{k}^{n+2 k-1}$ as additional hypotheses.

We next show that the Kneser-Lovász theorem (Theorem 1) follows from the truncated Tucker lemma on $\mathcal{B}_{k}^{n}$.

Proof of Theorem 1 from Theorem 22. Suppose for sake of contradiction that $c:\left(\begin{array}{l}n \\ k\end{array}\right) \rightarrow\{2 k, \ldots, n\}$ is an $(n-2 k+1)$-coloring of $\left(\begin{array}{l}n \\ k\end{array}\right)$. Let $\leq$ be a total order on $\left(\begin{array}{l}n \\ k\end{array}\right) \cup\{\emptyset\}$ that refines the partial order $\preceq$. Let $(A, B) \in \mathcal{B}_{k}^{n}$. Define $\lambda(A, B)$ as follows:

$$
\lambda(A, B)= \begin{cases}c(A) & \text { if } A>B \\ -c(B) & \text { if } B>A\end{cases}
$$

The map $\lambda$ is clearly antipodal, so by Theorem 22 , there is a pair $\left(A_{1}, B_{1}\right) \preceq\left(A_{2}, B_{2}\right) \in \mathcal{B}_{k}^{n}$ that is $k$-complementary. Since $\lambda$ must assign $\left(A_{1}, B_{1}\right)$ and $\left(A_{2}, B_{2}\right)$ opposite signs, it must be that either $A_{1}<B_{1} \leq B_{2}<A_{2}$ or $B_{1}<A_{1} \leq A_{2}<B_{2}$. In the former case, $c\left(B_{1}\right)=c\left(A_{2}\right)$ and in the latter case $c\left(A_{1}\right)=c\left(B_{2}\right)$. Since $B_{1} \cap A_{2}=A_{1} \cap B_{2}=\emptyset$, in either case we have a contradiction.

We claim that, for fixed $k$, the above proof of the Kneser-Lovász theorem from the truncated Tucker lemma can be translated into polynomial size constant depth Frege proofs. Recall that the propositional translations of the Kneser-Lovász theorem (see Definition 3) used propositional variables $p_{S, i}$; by inspection, they are polynomial size and constant depth. The propositional translations of the truncated Tucker lemma given above use variables $p_{A, B, i}$, and are also polynomial size and constant depth. Constant depth Frege proofs of the propositional Kneser-Lovász theorem from instances of the truncated Tucker lemma are formed as follows. For $A \neq B \in\left(\begin{array}{l}n \\ k\end{array}\right)$ and $i \in\{ \pm 2 k, \ldots, \pm n\}$, define the formula $\varphi_{A, B, i}$ to be $p_{A, i-2 k+1}$ if $A>B$ and $i>0$, to be $p_{B,-i-2 k+1}$ if $B>A$ and $i<0$, and to be the constant False otherwise. The "-2k+1" 
adjusts colors back to the range $[m]=[n-2 k+1]$, so this mimics the definition of $\lambda$ in (6), and the formulas $\varphi_{A, B, i}$ define a substitution instance of the propositional truncated Tucker lemma. The Kneser-Lovász theorem for the variables $p_{A, i}$ follows immediately from this instance of the truncated Tucker lemma by a simple case analysis.

\subsection{Equivalence between the truncated Tucker lemmas}

Theorem 23. The truncated Tucker lemma on $\mathcal{B}_{k}^{n}$ implies the truncated Tucker lemma on $\mathcal{B}_{\leq k}^{n-2 k+1}$.

Proof. Let $1<2 k \leq n$. Suppose that $\lambda: \mathcal{B}_{\leq k}^{n-2 k+1} \rightarrow\{1, \pm 2, \ldots, \pm(n-2 k+1)\}$ is an antipodal map. By renaming the range elements, we can instead write $\lambda: \mathcal{B}_{\leq k}^{n-2 k+1} \rightarrow\{1, \pm 2 k, \ldots, \pm(n-1)\}$. We will define $\lambda^{\prime}: \mathcal{B}_{k}^{n} \rightarrow\{ \pm 2 k, \ldots, \pm n\}$ as follows: For $(A, B) \in \mathcal{B}_{k}^{n}$,

$$
\lambda^{\prime}(A, B)= \begin{cases}\lambda\left(A^{*}, B^{*}\right) & \text { if } A \neq \emptyset \text { and } B \neq \emptyset \\ n & \text { if } A=\emptyset \\ -n & \text { if } B=\varnothing\end{cases}
$$

where $A^{*}=\{a \in A: a \leq n-2 k+1\}$. For $(A, B) \in \mathcal{B}_{k}^{n}$, we clearly have $\left(A^{*}, B^{*}\right) \in \mathcal{B}_{\leq k}^{n-2 k+1}$. We also claim that $\lambda^{\prime}(A, B)$ is never equal to 1 . To prove this, suppose $\lambda^{\prime}(A, B)=1$. By the definition of $\lambda^{\prime}$, both $A$ and $B$ are nonempty. Thus $\lambda\left(A^{*}, B^{*}\right)=1$ and consequently $A^{*}=B^{*}=\emptyset$. This means that $A$ and $B$ are both subsets of $\{n-2 k+2, \ldots, n\}$, a set of cardinality $2 k-1$. But this contradicts $A \cap B=\emptyset$ and $|A|=|B|=k$.

The map $\lambda^{\prime}$ is clearly antipodal by definition. By the truncated Tucker lemma on $\mathcal{B}_{k}^{n}$, there are pairs $\left(A_{1}, B_{1}\right) \preceq\left(A_{2}, B_{2}\right) \in$ $\mathcal{B}_{k}^{n}$ such that $\lambda^{\prime}\left(A_{1}, B_{1}\right)=-\lambda^{\prime}\left(A_{2}, B_{2}\right)$. We claim that $\lambda\left(A_{1}, B_{1}\right) \neq n$. Otherwise, $\lambda\left(A_{2}, B_{2}\right)=-n$, so $A_{1}=\emptyset$ and $B_{2}=\emptyset$, and this contradicts $\left(A_{1}, B_{1}\right) \preceq\left(A_{2}, B_{2}\right)$. Similarly, $\lambda\left(A_{1}, B_{1}\right) \neq-n$. It follows that all four sets $A_{1}, B_{1}, A_{2}, B_{2}$ are nonempty. Therefore, by the choice of $\left(A_{1}, B_{1}\right)$ and $\left(A_{2}, B_{2}\right)$,

$$
\lambda\left(A_{1}^{*}, B_{1}^{*}\right)=-\lambda\left(A_{2}^{*}, B_{2}^{*}\right) .
$$

We now claim that $\left(A_{1}^{*}, B_{1}^{*}\right) \preceq\left(A_{2}^{*}, B_{2}^{*}\right)$. Since $A_{1} \cap B_{2}=\emptyset$ and $A_{2} \cap B_{1}=\emptyset$, we have $A_{1}^{*} \cap B_{2}^{*}=\emptyset$ and $A_{2}^{*} \cap B_{1}^{*}=\emptyset$. Also, since $A_{1} \preceq A_{2}$,

$$
\left(A_{1} \cup A_{2}\right)_{\leq k}=A_{2}
$$

From this we obtain

$$
\left(A_{1}^{*} \cup A_{2}^{*}\right)_{\leq k}=\left(\left(A_{1} \cup A_{2}\right)_{\leq k}\right)^{*}=A_{2}^{*} .
$$

Thus $A_{1}^{*} \preceq A_{2}^{*}$. The same argument shows $B_{1}^{*} \preceq B_{2}^{*}$. This establishes that $\left(A_{1}^{*}, B_{1}^{*}\right)$ and $\left(A_{2}^{*}, B_{2}^{*}\right)$ are $k$-complementary with respect to $\lambda$.

The proof of Theorem 23 can be translated into polynomial size, constant depth Frege proofs. In other words, the propositional translations of the truncated Tucker lemma on $\mathcal{B}_{\leq k}^{n}$ can be proved with polynomial size, constant depth Frege proofs if all instances of the truncated Tucker lemma on $\mathcal{B}_{k}^{n}$ are given as additional hypotheses. This is proved analogously to the fact that the proof of Theorem 22 (truncated Tucker on $\mathcal{B}_{k}^{n}$ ) from instances of Theorem 20 (truncated Tucker on $\mathcal{B}_{\leq k}^{n}$ ) can be translated into constant depth Frege proofs. This establishes:

Corollary 24. The propositional translations of the truncated Tucker lemma on $\mathcal{B}_{k}^{n}$ have (quasi-)polynomial size Frege proofs if and only if the same holds for the truncated Tucker lemma on $\mathcal{B}_{\leq k}^{n}$.

\section{Short $e \mathcal{F}$ proofs of the truncated Tucker lemma, $k=1$ case}

In this section we prove the $k=1$ case of the truncated Tucker lemma. As we outline at the end of the paper, the argument is formalizable as polynomial size extended Frege proofs. Note that when $k=1$ the two versions of the truncated Tucker lemma are equivalent.

Recall the partial order $\preceq$ of Definition 17. When $k=1$, this partial order is a total order where $\{i\} \preceq\{j\}$ iff $i \geq j$. Thus,

$$
\emptyset \preceq\{n\} \preceq\{n-1\} \preceq \cdots \preceq\{2\} \preceq\{1\}
$$

is a complete description of $\preceq$ on $\left(\begin{array}{l}n \\ 1\end{array}\right)$.

Theorem 25. The $k=1$ case of the truncated Tucker lemma, Tucker ${ }_{1}^{n}$, has polynomial size extended Frege proofs. 
The polynomial size extended Frege proofs of the $k=1$ case of the truncated Tucker lemma are formed by formalizing the argument of Lemma 26 below.

Lemma 26. Suppose $n>2$ and $\lambda: \mathcal{B}_{1}^{n} \rightarrow\{ \pm 2, \ldots, \pm n\}$ is an antipodal map with no 1-complementary pairs. Then there is an antipodal map $\lambda^{\prime}: \mathcal{B}_{1}^{n-1} \rightarrow\{ \pm 2, \ldots, \pm(n-1)\}$ with no 1-complementary pairs.

Proof. Given $\lambda$ satisfying the hypothesis, let $\ell=\lambda(\{n\}, \emptyset)$. We will define an antipodal map $\lambda^{\prime}: \mathcal{B}_{1}^{n-1} \rightarrow\{ \pm 2, \ldots, \pm n\} \backslash\{ \pm \ell\}$. Let $(A, B) \in \mathcal{B}_{1}^{n-1}$. The value $\lambda^{\prime}(A, B)$ will be defined by cases:

Case 1: If $(A, B) \in \mathcal{B}_{1}^{n-1}$ with $|A|=|B|=1$, then $\lambda^{\prime}(A, B)=\lambda(A, B)$.

Case 2: If $(A, \emptyset) \in \mathcal{B}_{1}^{n-1}$, then $\lambda^{\prime}(A, \emptyset)$ is defined by cases:

Case 2a: If $\ell \notin\{\lambda(X, \emptyset):\{n-1\} \preceq X \preceq A\}$, then define $\lambda^{\prime}(A, \emptyset)$ to be $\lambda(A, \emptyset)$.

Case $2 \mathrm{~b}$ : If case $2 \mathrm{a}$ does not apply, then define $\lambda^{\prime}(A, \emptyset)$ to be $\lambda(A,\{n\})$.

Case 3: If $(\emptyset, B) \in \mathcal{B}_{1}^{n-1}$, then $\lambda^{\prime}(\emptyset, B)$ is defined to be $-\lambda^{\prime}(B, \emptyset)$, where $\lambda^{\prime}(B, \emptyset)$ has already been defined by case 2 .

The map $\lambda^{\prime}$ is antipodal because $\lambda$ is.

Claim 27. The map $\lambda^{\prime}$ never maps to $\ell$ or $-\ell$.

The argument splits into cases:

- Suppose $(A, B) \in \mathcal{B}_{1}^{n-1}$, with $|A|=|B|=1$. Then $\lambda^{\prime}(A, B)=\lambda(A, B)$. Since $|A|=1,\{n\} \preceq A$, and since $B \in\left(\begin{array}{c}n-1 \\ 1\end{array}\right)$, it follows that $\{n\} \cap B=\emptyset$. Additionally $\emptyset \preceq B$, and $\emptyset \cap A=\emptyset$. Therefore $(\{n\}, \emptyset) \preceq(A, B)$. Since $\lambda$ has no 1-complementary pairs, and $\lambda(\{n\}, \emptyset)=\ell$, it follows that $\lambda(A, B) \neq-\ell$. Therefore $\lambda^{\prime}(A, B) \neq-\ell$. Because $\lambda^{\prime}$ is antipodal, this also proves $\lambda^{\prime}(A, B) \neq \ell$.

- Suppose $(A, \emptyset) \in \mathcal{B}_{1}^{n-1}$, and $\lambda^{\prime}(A, \emptyset)$ is assigned by case 2a. For case 2a to apply, it must be that $\lambda(A, \emptyset) \neq \ell$. Furthermore, $\lambda(\{n\}, \emptyset)=\ell,(\{n\}, \emptyset) \preceq(A, \emptyset)$, and the fact that $\lambda$ has no 1-complementary pairs imply that $\lambda(A, \emptyset) \neq-\ell$. Therefore, $\lambda^{\prime}(A, \emptyset)=\lambda(A, \emptyset) \neq \pm \ell$.

- Suppose $(A, \emptyset) \in \mathcal{B}_{1}^{n-1}$, and $\lambda^{\prime}(A, \emptyset)$ is assigned by case $2 \mathrm{~b}$. This implies that there is some $X \in\left(\begin{array}{c}n-1 \\ 1\end{array}\right)$ with $\{n-1\} \preceq$ $X \preceq A$ such that $\lambda(X, \emptyset)=\ell$. Note that $\{n-1\} \preceq X$ implies that $\{n\} \cap X=\emptyset$. Since $(X, \emptyset) \preceq(A$, \{n\}), it follows that $\lambda(A,\{n\}) \neq-\ell$. Since $\lambda(\emptyset,\{n\})=-\ell$ and $(\emptyset,\{n\}) \preceq(A,\{n\})$ it follows that $\lambda(A,\{n\}) \neq \ell$. Thus $\lambda^{\prime}(A, \emptyset)=\lambda(A,\{n\}) \neq \pm \ell$.

- Suppose $(\emptyset, B) \in \mathcal{B}_{1}^{n-1}$. Then $\lambda^{\prime}(\emptyset, B)=-\lambda^{\prime}(B, \emptyset)$, and we have shown above that $\lambda^{\prime}(B, \emptyset) \neq \pm \ell$.

This completes the proof of Claim 27.

Claim 28. The map $\lambda^{\prime}$ has no 1-complementary pairs.

We show the contrapositive. The argument splits into cases:

- Suppose $\left(A_{1}, B_{1}\right) \preceq\left(A_{2}, B_{2}\right) \in \mathcal{B}_{1}^{n-1}$ with $\left|A_{1}\right|=\left|B_{1}\right|=\left|A_{2}\right|=\left|B_{2}\right|=1$. Then $\lambda^{\prime}\left(A_{1}, B_{1}\right)$ and $\lambda^{\prime}\left(A_{2}, B_{2}\right)$ both are assigned by case 1 . Thus,

$$
\lambda\left(A_{1}, B_{1}\right)=\lambda^{\prime}\left(A_{1}, B_{1}\right)=-\lambda^{\prime}\left(A_{2}, B_{2}\right)=-\lambda\left(A_{2}, B_{2}\right)
$$

Therefore $\lambda$ has a 1-complementary pair.

- Suppose $\left(A_{1}, \emptyset\right) \preceq\left(A_{2}, B_{2}\right) \in \mathcal{B}_{1}^{n-1}$, with $\lambda^{\prime}\left(A_{1}, \emptyset\right)$ assigned by case 2 a and $\lambda^{\prime}\left(A_{2}, B_{2}\right)$ assigned by case 1 . So $\lambda\left(A_{1}, \emptyset\right)=$ $-\lambda\left(A_{2}, B_{2}\right)$. Thus $\lambda$ has a 1 -complementary pair.

- Suppose $\left(A_{1}, \emptyset\right) \preceq\left(A_{2}, B_{2}\right) \in \mathcal{B}_{1}^{n-1}$, with $\lambda^{\prime}\left(A_{1}, \emptyset\right)$ assigned by case $2 \mathrm{~b}$ and $\lambda^{\prime}\left(A_{2}, B_{2}\right)$ assigned by case 1 . So $\lambda\left(A_{1},\{n\}\right)=$ $-\lambda\left(A_{2}, B_{2}\right)$. Since $\left(A_{1},\{n\}\right) \preceq\left(A_{2}, B_{2}\right)$, it follows that $\lambda$ has a 1-complementary pair.

- Suppose $\left(A_{1}, \emptyset\right) \preceq\left(A_{2}, \emptyset\right) \in \mathcal{B}_{1}^{n-1}$, with $\lambda^{\prime}\left(A_{1}, \emptyset\right)$ and $\lambda^{\prime}\left(A_{2}, \emptyset\right)$ both assigned by case 2a. So then $\lambda\left(A_{1}, \emptyset\right)=-\lambda\left(A_{2}, \emptyset\right)$, hence $\lambda$ has a 1-complementary pair.

- Suppose $\left(A_{1}, \emptyset\right) \preceq\left(A_{2}, \emptyset\right) \in \mathcal{B}_{1}^{n-1}$, with $\lambda^{\prime}\left(A_{1}, \emptyset\right)$ and $\lambda^{\prime}\left(A_{2}, \emptyset\right)$ both assigned by case 2 b. So then $\lambda\left(A_{1},\{n\}\right)=$ $-\lambda\left(A_{2},\{n\}\right)$, hence $\lambda$ has a 1-complementary pair.

- Suppose $\left(A_{1}, \emptyset\right) \preceq\left(A_{2}, \emptyset\right) \in \mathcal{B}_{1}^{n-1}$, with $\lambda^{\prime}\left(A_{1}, \emptyset\right)$ assigned by case $2 \mathrm{a}$ and $\lambda^{\prime}\left(A_{2}, \emptyset\right)$ assigned by case $2 \mathrm{~b}$. Thus,

$$
\lambda\left(A_{1}, \emptyset\right)=\lambda^{\prime}\left(A_{1}, B_{1}\right)=-\lambda^{\prime}\left(A_{2}, B_{2}\right)=-\lambda\left(A_{2},\{n\}\right)
$$

and since $\left(A_{1}, \emptyset\right) \preceq\left(A_{2},\{n\}\right)$, it follows that $\lambda$ has a 1-complementary pair. 
- Suppose $\left(A_{1}, B_{1}\right) \preceq\left(A_{2}, \emptyset\right) \in \mathcal{B}_{1}^{n-1}$ where $\left|A_{1}\right|=\left|B_{1}\right|=1$. This impossible, because $B_{1} \preceq \emptyset$, and no set of cardinality 1 precedes the emptyset under the partial order $\preceq$.

- Suppose $\left(A_{1}, \emptyset\right) \preceq\left(A_{2}, \emptyset\right) \in \mathcal{B}_{1}^{n-1}$, and $\lambda^{\prime}\left(A_{1}, \emptyset\right)$ is assigned by case $2 \mathrm{~b}$ and $\lambda^{\prime}\left(A_{2}, \emptyset\right)$ is assigned by case $2 \mathrm{a}$. Then there exists an $X \in\left(\begin{array}{c}n-1 \\ 1\end{array}\right)$ such that $\{n-1\} \preceq X \preceq A_{1}$ and $\lambda(X, \emptyset)=\ell$. Since $A_{1} \preceq A_{2}$, it follows that $\{n-1\} \preceq X \preceq A_{2}$. This implies that $\lambda^{\prime}\left(A_{2}, \emptyset\right)$ is not assigned by case 2 a, so this case is impossible.

- Suppose $\left(A_{1}, \emptyset\right) \preceq\left(\emptyset, B_{2}\right) \in \mathcal{B}_{1}^{n-1}$. This is impossible, because $A_{1} \preceq \emptyset$ implies that $A_{1}=\emptyset$, but $(\emptyset, \emptyset) \notin \mathcal{B}_{1}^{n-1}$.

- The remaining cases involving case 3 of the definition of $\lambda^{\prime}$ follow from above, using the fact that if $\left(A_{1}, B_{1}\right) \preceq\left(A_{2}, B_{2}\right)$ form a 1-complementary pair, then $\left(B_{1}, A_{1}\right) \preceq\left(B_{2}, A_{2}\right)$ also form a 1-complementary pair.

This completes the proof of Claim 28. Claims 27 and 28 suffice to prove Lemma 26.

We are now ready to sketch the proof of polynomial size extended Frege proofs of Tucker $_{1}^{n}$.

Proof of Theorem 25. To give an extended Frege proof of $\operatorname{Tucker}_{1}^{n}(\vec{p})$, where $\vec{p}$ is a set of propositional variables encoding a map $\lambda$, we use the extension rule to introduce new variables $\vec{p}^{\prime}$ to encode $\lambda^{\prime}$ as in Lemma 26. It is straightforward to see that the definition of $\lambda^{\prime}$ from $\lambda$ can be carried out by polynomial size formulas. Furthermore, there are polynomial size proofs of $\neg \operatorname{Tucker}_{1}^{n}(\vec{p}) \rightarrow \neg \operatorname{Tucker}_{1}^{n-1}\left(\vec{p}^{\prime}\right)$, namely they formalize the argument of Lemma 26 . This process is repeated, using the extension rule to introduce new propositional variables each round, until the proof reaches $\neg \operatorname{Tucker}_{1}^{2}\left(\vec{p}^{\prime \prime}\right)$. From here, the extended Frege proof concludes with a constant size proof of $\operatorname{Tucker}_{1}^{2}\left(\vec{p}^{\prime \prime}\right)$.

Question 29. Do the propositional translations of the truncated Tucker lemma for $k>1$ have short (extended) Frege proofs?

\section{References}

[1] J. Aisenberg, M.L. Bonet, S. Buss, Tucker's lemma is PPA complete, 2015, submitted for publication.

[2] J. Aisenberg, M.L. Bonet, S. Buss, Quasi-polynomial size Frege proofs of Frankl's theorem on the trace of finite sets, J. Symb. Log. 81 (2) (2016) 1-24.

[3] J. Aisenberg, M.L. Bonet, S. Buss, A. Crăciun, G. Istrate, Short proofs of the Kneser-Lovász coloring principle, in: Proc. 42th International Colloquium on Automata, Languages, and Programming, ICALP'15, in: Lecture Notes in Computer Science, vol. 9135, 2015, pp. 44-55.

[4] J. Avigad, Plausibly hard combinatorial tautologies, in: P. Beame, S.R. Buss (Eds.), Proof Complexity and Feasible Arithmetics, American Mathematical Society, 1997, pp. 1-12.

[5] A. Beckmann, S.R. Buss, Improved witnessing and local improvement principles for second-order bounded arithmetic, ACM Trans. Comput. Log. 15 (1) (2014) 2.

[6] M.L. Bonet, S.R. Buss, T. Pitassi, Are there hard examples for Frege systems?, in: P. Clote, J. Remmel (Eds.), Feasible Mathematics II, Birkhäuser, Boston, 1995, pp. 30-56.

[7] S. Buss, Quasipolynomial size proofs of the propositional pigeonhole principle, Theor. Comput. Sci. 576 (C) (2015) 77-84.

[8] S.R. Buss, Bounded Arithmetic, Bibliopolis, revision of 1985 Princeton University Ph.D. thesis, 1986.

[9] S.R. Buss, Polynomial size proofs of the propositional pigeonhole principle, J. Symb. Log. 52 (1987) 916-927.

[10] S.R. Buss, Propositional consistency proofs, Ann. Pure Appl. Log. 52 (1991) 3-29.

[11] S.R. Buss, Propositional proof complexity: an introduction, in: U. Berger, H. Schwichtenberg (Eds.), Computational Logic, Springer-Verlag, Berlin, 1999, pp. 127-178.

[12] S.R. Buss, Towards NP-P via proof complexity and proof search, Ann. Pure Appl. Log. 163 (9) (2012) 1163-1182.

[13] S.A. Cook, Feasibly constructive proofs and the propositional calculus, in: Proceedings of the Seventh Annual ACM Symposium on Theory of Computing, 1975, pp. 83-97.

[14] S.A. Cook, R.A. Reckhow, On the lengths of proofs in the propositional calculus, preliminary version, in: Proceedings of the Sixth Annual ACM Symposium on the Theory of Computing, 1974, pp. 135-148.

[15] S.A. Cook, R.A. Reckhow, The relative efficiency of propositional proof systems, J. Symb. Log. 44 (1979) 36-50.

[16] P. Erdős, C. Ko, R. Rado, Intersection theorems for systems of finite sets, Q. J. Math., Oxford Second Ser. 12 (1961) 313-320.

[17] P. Frankl, Z. Füredi, Non-trivial intersecting families, J. Comb. Theory, Ser. A 41 (1) (1986) 150-153.

[18] R.M. Freund, M.J. Todd, A constructive proof of Tucker's combinatorial lemma, J. Comb. Theory, Ser. A 30 (1981) 321-325.

[19] A.J.W. Hilton, E.C. Milner, Some intersection theorems for systems of finite sets, Q. J. Math., Oxford Second Ser. 18 (1967) 369-384.

[20] P. Hrubeš, I. Tzameret, Short proofs for determinant identities, SIAM J. Comput. 44 (2) (2015) 340-383.

[21] G. Istrate, A. Crăciun, Proof complexity and the Kneser-Lovász theorem, in: Theory and Applications of Satisfiability Testing, SAT, in: Lecture Notes in Computer Science, vol. 8561, Springer-Verlag, 2014, pp. 138-153.

[22] E. Jeřábek, Dual weak pigeonhole principle, Boolean complexity, and derandomization, Ann. Pure Appl. Log. 124 (2004) 1-37.

[23] L.A. Kołodziejczyk, P. Nguyen, N. Thapen, The provably total NP search problems of weak second-order bounded arithmetic, Ann. Pure Appl. Log. 162 (2) (2011) 419-446.

[24] J. Krajičcek, Bounded Arithmetic, Propositional Calculus and Complexity Theory, Cambridge University Press, Heidelberg, 1995.

[25] L. Lovász, Kneser's conjecture, chromatic number, and homotopy, J. Comb. Theory, Ser. A 25 (3) (1978) 319-324.

[26] J. Matoušek, A combinatorial proof of Kneser's conjecture, Combinatorica 24 (1) (2004) 163-170.

[27] C.H. Papadimitriou, On the complexity of the parity argument and other inefficient proofs of existence, J. Comput. Syst. Sci. 48 (3) (1994) $498-532$.

[28] P. Pudlák, On the lengths of proofs of finitistic consistency statements in first order theories, in: Logic Colloquium '84, North-Holland, 1986, pp. $165-196$.

[29] P. Pudlák, Improved bounds to the lengths of proofs of finitistic consistency statements, in: Logic and Combinatorics, in: Contemporary Mathematics, vol. 65, American Mathematical Society, 1987, pp. 309-331.

[30] N. Segerlind, The complexity of propositional proofs, Bull. Symb. Log. 13 (4) (2007) 417-481.

[31] R. Statman, Complexity of derivations from quantifier-free Horn formulae, mechanical introduction of explicit definitions, and refinement of completeness theorems, in: R. Gandy, M. Hyland (Eds.), Logic Colloquium '76, North-Holland, Amsterdam, 1977, pp. 505-517.

[32] G.M. Ziegler, Generalized Kneser coloring theorems with combinatorial proofs, Invent. Math. 147 (3) (2002) 671-691. 


\section{Sponsor names}

Do not correct this page. Please mark corrections to sponsor names and grant numbers in the main text.

NSF, country=United States, grants=DMS $-1101228, \quad \mathrm{CCF}-1213151$

Simons Foundation, country=United States, grants $=306202$

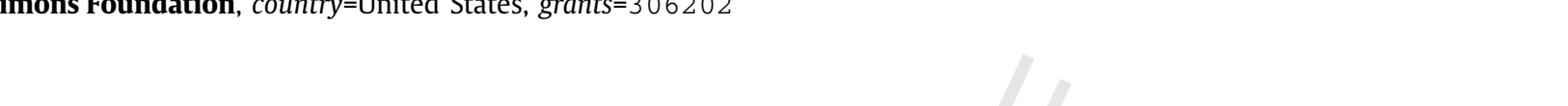

